\title{
Non-thermal cosmic backgrounds from blazars: the contribution to the CMB, X-ray and $\gamma$-ray backgrounds ${ }^{\star}$
}

\author{
P. Giommi ${ }^{1,2}$, S. Colafrancesco ${ }^{3}$, E. Cavazzuti ${ }^{1,2}$, M. Perri ${ }^{1}$, and C. Pittori ${ }^{1,4}$ \\ 1 ASI Science Data Center, ASDC c/o ESRIN, via G. Galilei, 00044 Frascati, Italy \\ e-mail: paolo.giommi@asi.it \\ 2 Agenzia Spaziale Italiana, Unitá Osservazione dell’Universo, viale Liegi 26, 00198 Roma, Italy \\ 3 INAF - Osservatorio Astronomico di Roma via Frascati 33, 00040 Monteporzio, Italy \\ 4 Universitá di Roma "Tor Vergata” and INFN sez. Roma 2, Italy
}

Received 11 May 2005 / Accepted 8 August 2005

\begin{abstract}
We present a new assessment of the contribution of the blazar population to the extragalactic background radiation across the electromagnetic spectrum. Our calculations rely on deep blazar radio counts that we have derived by combining several radio and multi-frequency surveys. We show that blazar emission integrated over cosmic time gives rise to a considerable broad-band non-thermal cosmic background that in some parts of the electromagnetic spectrum dominates the extragalactic brightness.

We confirm that blazars are the main discrete contributors to the Cosmic Microwave Background (CMB), where we estimate that their integrated emission causes an apparent temperature increase of 5-50 $\mu \mathrm{K}$ in the frequency range 50-250 GHz. The CMB primordial fluctuation spectrum is contaminated starting at multipole $l \approx 300-600$, in the case of a completely random source distribution, or at lower $l$ values if spatial clustering is present. We estimate that well over one hundred-thousand blazars will produce a significant signal in the maps of the upcoming Planck CMB anisotropy mission. Because of a tight correlation between the microwave and the X-ray flux, these sources are expected to be $\mathrm{X}$-ray emitters with flux larger than a few $10^{-15} \mathrm{erg} \mathrm{cm}^{-2} \mathrm{~s}^{-1}$ in the soft X-ray band. A large fraction of the foreground sources in current and near-future CMB anisotropy maps could therefore be identified and removed using a multi-frequency approach, provided that a sufficiently deep all-sky X-ray survey will become available in the near future.

We show further that blazars are a major constituent of all high energy extragalactic backgrounds. Their contribution is expected to be $11-12 \%$ at X-ray frequencies and possibly $100 \%$ in the $\sim 0.5-50 \mathrm{MeV}$ band. At higher energies $(E>100 \mathrm{MeV})$ the estimated blazar collective emission, obtained by extrapolating their integrated micro-wave flux to the $\gamma$-ray band using the SED of EGRET detected sources, overestimates the extragalactic background by a large factor, thus implying that not only blazars dominate the $\gamma$-ray sky but also that their average duty cycle at these frequencies must be rather low. Finally, we find that blazars of the HBL type may produce a significant amount of flux at TeV energies.
\end{abstract}

Key words. radiation mechanisms: non-thermal - BL Lacertae objects: general - quasars: general

\section{Introduction}

The identification of the first quasar (Schmidt 1963) marked the beginning of not only AGN astrophysics but also the discovery of the first flat spectrum radio quasar (3C 273), a type of highly variable, often polarized extragalactic radio source that, together with the even more puzzling BL Lacertae objects, makes the class of blazars, the most extreme type of AGN known. The blazar's typical observational properties include the emission of electromagnetic radiation across the entire spectrum from radio waves to the most energetic $\gamma$-rays, irregular rapid variability, apparent super-luminal motion, flat radio spectrum, large and variable polarization at radio and especially, at optical frequencies.

$\star$ Table 4 is only available in electronic form at http://www. edpsciences.org
Blazars of the BL Lacertae type (BL Lacs) are distinguished by non-thermal emission with no (or very weak) emission lines, are often associated with the nuclei of elliptical galaxies, and are the only population of extragalactic sources that shows negative cosmological evolution (Bade et al. 1998; Giommi et al. 1999; Rector et al. 2000). Flat Spectrum Radio Quasars (FSRQs) share the strong non-thermal emission of BL Lacs, but also show intense broad line emission and strong cosmological evolution similar to that of radio quiet QSOs (Caccianiga et al. 2002; Landt et al. 2001; Wall \& Peacock 1985).

Blazars are widely assumed to be powerful sources that emit a continuum of electromagnetic (e.m.) radiation from a relativistic jet viewed closely along the line of sight (Blandford \& Rees 1978; Urry \& Padovani 1995). The broad-band electromagnetic spectrum is composed of a synchrotron low-energy 


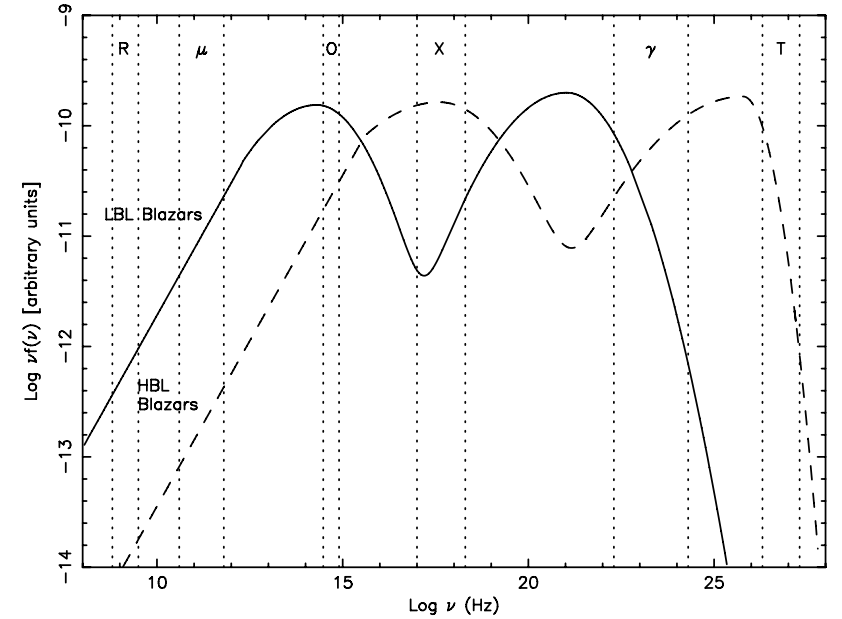

Fig. 1. The Spectral Energy Distribution of different types of Blazars represented by Synchrotron-Self-Compton models with emission peaking at different energies. LBL sources are those where the synchrotron peak is located at Infra-Red frequencies and therefore their $\mathrm{X}$-ray emission is due to Inverse Compton radiation. HBL Blazars are those where the synchrotron component peaks in the UV/X-ray band making their $f_{\mathrm{x}} / f_{\mathrm{r}}$ flux ratio hundreds or thousands of times higher than that of LBLs.

component that peaks (in a $\log (v f(v))-\log (v)$ representation) between the far infrared and the X-ray band, followed by an Inverse Compton component that has its maximum in the hard $\mathrm{X}$-ray band or at higher energies, depending on the location of the synchrotron peak, and extends into the $\gamma$-ray or even the $\mathrm{TeV}$ band. Those blazars where the synchrotron peak is located at low energy are usually called Low energy peaked blazars or LBL, while those where the synchrotron component reaches the X-ray band are called High energy peaked blazars or HBL (see Fig. 1 and Padovani \& Giommi 1995). LBL sources are the large majority among blazars (e.g., Padovani et al. 2003a) and are usually discovered in radio surveys, while HBL objects are preferentially found in X-ray flux limited surveys, since at these frequencies they are hundreds, or even thousands, of times brighter than LBLs of similar radio power.

Radio-quiet AGN are approximately one order of magnitude more abundant than blazars and have been shown to be the major constituent of the Cosmic X-ray Background (CXB) (Giacconi et al. 1962; Rosati et al. 2002; Moretti et al. 2003), leading to the now consolidated picture in which the CXB is composed of radiation generated by the accretion onto supermassive black holes integrated over cosmic time.

Despite their relatively low space density, blazars' strong emission across the entire electromagnetic spectrum makes them potential candidates as significant contributors to extragalactic cosmic backgrounds at frequencies where the accretion mechanism does not produce much radiation. These extragalactic backgrounds would then be composed mostly of non-thermal radiation generated in Synchrotron/Inverse Compton-type environments.

Recently, Giommi \& Colafrancesco (2004) showed that blazars are by far the largest population of extragalactic objects detected as foreground sources in CMB anisotropy maps and

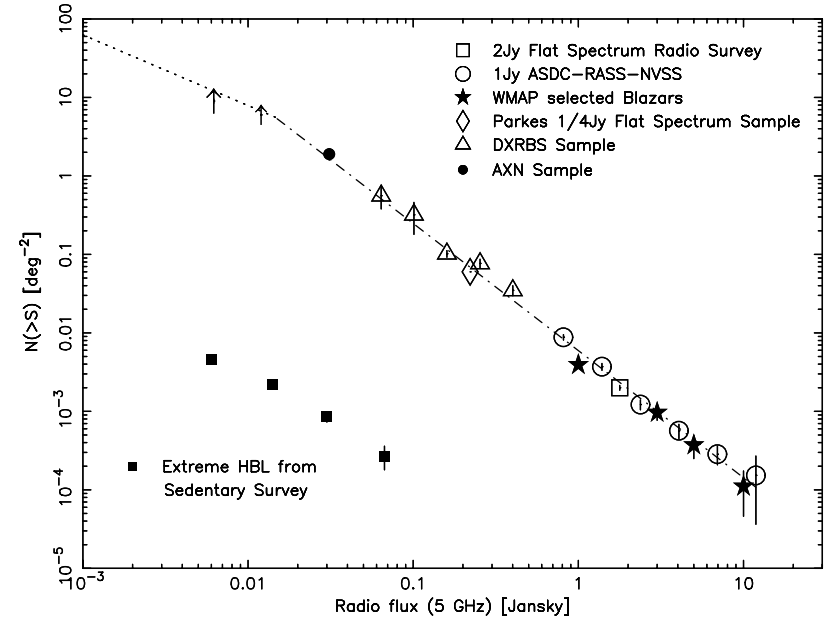

Fig. 2. The radio $(5 \mathrm{GHz}) \log N-\log S$ of Blazars built combining several radio and multi-frequency surveys. The different symbols represent the surveys used (see text for details). The filled square symbols in the lower part of the diagram represent the $\log N-\log S$ of extreme HBL BL Lacs from the Sedentary Survey. Although these objects are a tiny minority $(\approx 0.1 \%)$ of the overall Blazar population they play an important role in producing the Cosmic Background at X-ray and at very high energies $(\gamma$-ray/TeV).

that their emission contaminates the $\mathrm{CMB}$ angular power spectrum to a significant level. Based on the detection of a small sample of blazars at $\gamma$-ray frequencies, Padovani et al. (1993) concluded that the blazar population should produce a large fraction of the high energy cosmic background.

In this paper we re-assess the blazar contribution to the Cosmic energy in the microwave (CMB), the X-ray (CXB), the Gamma-ray (CGB) and TeV (CTB) part of the electromagnetic spectrum. Our calculations rely on a new estimation of the blazar radio $\log N-\log S$, that we have assembled by combining several radio and multi-frequency surveys, on flux ratios in different energy bands, and on observed blazar broad-band Spectral Energy Distributions (SED).

\section{The blazar radio $\log N$ - Log $S$}

We used several radio and multi-frequency surveys to build a new deep blazar radio $\log N-\log S$ that updates and extends the counts presented in Giommi \& Colafrancesco (2004) to lower fluxes. Since these surveys were carried out at three different observing frequencies $(1.4,2.7$ and $5 \mathrm{GHz})$, we converted all flux densities to a common frequency before proceeding. We took $5 \mathrm{GHz}$ as the reference frequency and, unless otherwise stated, we applied the flux conversions by assuming a spectral slope $\alpha_{\mathrm{r}}=0.25\left(f \propto v^{-\alpha_{\mathrm{r}}}\right)$ that is approximately equal to the average value in all our samples.

The derived blazar counts are shown in Fig. 2 and can be described by a broken power law with parameters defined in the following equation:

$N(>S)= \begin{cases}5.9510^{-3} \times S^{-1.62} & S>0.015 \mathrm{Jy} \\ 0.125 \times S^{-0.9} & S<0.015 \mathrm{Jy} .\end{cases}$

The slope up to $15 \mathrm{mJy}$ (dot-dashed line) is a good representation of the data, whereas the flattening at fainter fluxes (dotted 
Table 1. One Jansky ASDC-RASS-NVSS sources.

\begin{tabular}{cl}
\hline \hline $\begin{array}{c}\text { Number } \\
\text { of sources }\end{array}$ & \multicolumn{1}{c}{ Classification } \\
\hline 132 & FSRQs \\
25 & BL Lac \\
36 & SSRQs \\
21 & radio galaxies \\
3 & other galaxies \\
9 & unidentified (3 flat, 6 steep) \\
\hline
\end{tabular}

line) is necessary to avoid the predicted blazar space density exceeding the total density of NVSS radio sources at a few mJy. The slope below the break is somewhat arbitrary as only upper limits are available in this flux regime. We chose a value of 0.9 since this is the average slope of the $\log N-\log S$ of radio quiet AGN in the two flux decades below the break (Rosati et al. 2002; Moretti et al. 2003) and is consistent with all the available constraints.

In the following, we discuss the details of all the surveys in order of decreasing radio flux limit.

\subsection{Two Jansky flat spectrum radio survey}

The Two Jansky $2.7 \mathrm{GHz}$ sample (Wall \& Peacock 1985) is based on a complete radio flux limited survey of flat spectrum $\left(\alpha_{\mathrm{r}}<0.5, f \propto v^{-\alpha_{\mathrm{r}}}\right)$ sources covering the entire sky with the exclusion of the Galactic plane $(|b|>10)$. The sample includes 60 blazars (di Serego-Alighieri et al. 1994; Urry \& Padovani 1995 ) corresponding to a space density of $0.002 \mathrm{deg}^{-2}$. This value has been plotted as an open square symbol in Fig. 2.

\subsection{One Jansky ASDC-RASS-NVSS blazar sample}

The ASDC-RASS-NVSS 1 Jy Blazar (1 Jy-ARN) Survey (Giommi et al. 2002c) is a radio flux limited $\left(f_{\mathrm{r}}>\right.$ 1 Jy@1.4 GHz) sample of blazars selected by means of a cross correlation between the ROSAT All Sky Survey (RASS) catalog of X-ray sources (Voges et al. 1999) and the subsample of NVSS survey (Condon et al. 1998) sources with flux density larger than 1 Jansky. The selection was carried out applying the same multi-frequency technique used for the definition of the extreme HBL BL Lac sample in the Sedentary survey (Giommi et al. 1999). To avoid the complications due to high $N_{\mathrm{H}}$ photoelectric absorption close to the Galactic plane, only sources located at Galactic latitudes higher than 20 degrees were considered. The accurate radio positions allowed us to obtain firm associations with all the optical counterparts. The sample includes a total of 226 sources, $96 \%$ of which were previously known objects, in most cases well-documented in the literature as they are bright radio sources. The class composition of the sample is reported in Table 1.

From past $\mathrm{X}$-ray measurements we know that blazars are characterized by a $f_{\mathrm{x}} / f_{\mathrm{r}}$ ratio ranging from $\approx 1 . \times$ $10^{-13} \mathrm{erg} \mathrm{cm}^{-2} \mathrm{~s}^{-1} \mathrm{Jy}^{-1}$ to over $1 . \times 10^{-9} \mathrm{erg} \mathrm{cm}^{-2} \mathrm{~s}^{-1} \mathrm{Jy}^{-1}$ (Padovani 2002); therefore all blazars with flux larger than

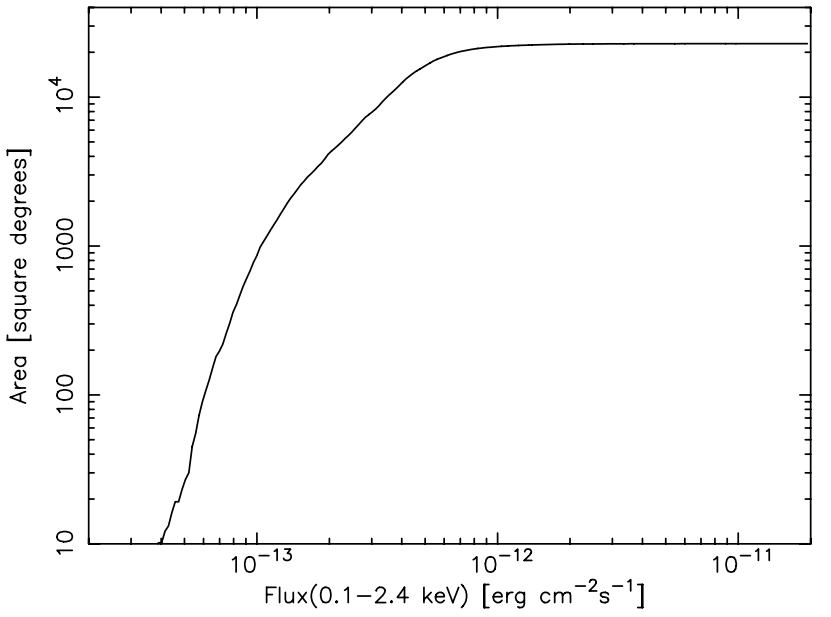

Fig. 3. The high Galactic Latitudes $(|b|>20)$ sky coverage of the RASS survey in the region of overlap with the NVSS catalog (Dec > -40 degrees).

1 Jy should be detectable in a survey with X-ray sensitivity of 1. $\times 10^{-13} \mathrm{erg} \mathrm{cm}^{-2} \mathrm{~s}^{-1} \mathrm{Jy}^{-1}$. However, the X-ray limiting flux of the RASS strongly varies across the sky depending on the effective exposure and on the amount of Galactic absorption $\left(N_{\mathrm{H}}\right)$ along the line of sight.

Figure 3 shows the high Galactic Latitude $(|b|>20)$ sky coverage of the RASS in the region of overlap with the NVSS survey. The curve was calculated taking the ROSAT effective exposure into account in sky bins of 1 square degree in size and was then converted to sensitivity assuming a power law spectrum with energy index $\alpha=1.0$ and setting the amount of $N_{\mathrm{H}}$ equal to the Galactic value in the direction of each sky bin.

The X-ray sensitivity ranges from somewhat below $1 \times$ $10^{-13} \mathrm{erg} \mathrm{cm}^{-2} \mathrm{~s}^{-1}$, where about 1000 square degrees of sky are covered, to $\approx 2 . \times 10^{-12} \mathrm{erg} \mathrm{cm}^{-2} \mathrm{~s}^{-1}$, where the maximum area of 23000 square degrees is reached.

Assuming that the three unidentified flat spectrum sources are blazars, the number of objects of this type (FSRQ+BL Lacs+unidentified sources with $\alpha_{\mathrm{r}}<0.5$ ) in the 1 Jy-ARN survey is 160 .

This sample was used to estimate the blazar space density above 1 Jy taking into account that the sky coverage of Fig. 3 implies that faint sources with flux around e.g. $10^{-13} \mathrm{erg} \mathrm{cm}^{-2} \mathrm{~s}^{-1}$ could be detected only in $\approx 1000$ square degrees of sky, whereas brighter X-ray sources with flux of e.g. $10^{-12} \mathrm{erg} \mathrm{cm}^{-2} \mathrm{~s}^{-1}$ could be detected over a much larger portion of the sky ( $\approx 20000$ square degrees). The counts were converted to $5 \mathrm{GHz}$ and then plotted as open circles in Fig. 2.

\subsection{WMAP microwave selected blazars}

The Wilkinson Microwave Anisotropy Probe (WMAP) is a space observatory dedicated to accurately investigating primordial fluctuations in the Cosmic Microwave Background (Bennett et al. 2003a). A catalog of 208 bright foreground sources detected in one or more of the five microwave WMAP channels during the first-year all-sky survey has been published 
Table 2. WMAP bright foreground source catalog.

\begin{tabular}{cl}
\hline \hline $\begin{array}{c}\text { Number } \\
\text { of sources }\end{array}$ & \multicolumn{1}{c}{ Classification } \\
\hline 141 & FSRQs \\
23 & BL Lac \\
13 & radio galaxies \\
5 & SSRQs \\
2 & starburst galaxies \\
2 & planetary nebulae \\
17 & unidentified \\
5 & no radio counterpart \\
& (probably spurious) \\
\hline
\end{tabular}

by Bennett et al. (2003b). With very few exceptions, all entries are well-known bright sources at $\mathrm{cm}$ wavelengths and in most cases were observed at several radio frequencies. Giommi \& Colafrancesco (2004) have recently shown that the large majority of these objects are blazars.

To determine how WMAP-selected blazars contribute to the $5 \mathrm{GHz}$ counts, we considered all high Galactic latitude $(|b|>20 \mathrm{deg})$ detections with flat radio spectrum $\left(\alpha_{\mathrm{r}}<0.5\right.$, $\left.f \propto v^{-\alpha_{\mathrm{r}}}\right)$ and with the SED typical of blazars. Table 2 gives detailed statistics.

The space density of WMAP detected sources with radio flux above 1, 3, 5, and 10 Jansky at $5 \mathrm{GHz}$ is plotted in Fig. 2 as filled-star symbols.

With the exception of the point at 1 Jansky, which is most likely an underestimation due to incompleteness of the WMAP catalog at this flux limit (Bennett et al. 2003b), the agreement with other radio surveys at $\mathrm{cm}$ wavelengths is very good, implying that the blazar selected by WMAP and at radio frequencies belong to the same population of objects.

\subsection{The Parkes quarter-Jansky Flat Spectrum sample}

The Parkes Quarter-Jansky Flat Spectrum sample (Wall et al. 2004 ) is a $100 \%$ identified radio flux limited survey at a frequency of $2.7 \mathrm{GHz}$.

A total of 328 FSRQ and 43 BL Lacs have been detected with flux density higher than $0.25 \mathrm{Jy}$ in an area of 8785 square degrees (sample 1 of Wall et al. 2004). Considering that this survey only accepts sources with spectral index flatter than $\alpha_{\mathrm{r}}<0.4\left(f \propto v^{-\alpha_{\mathrm{r}}}\right)$, we rescale the sample density by a factor $1 / 0.75$, which is the ratio of QSOs and BL Lacs with $\alpha_{\mathrm{r}}<0.7$ to those with $\alpha_{\mathrm{r}}<0.4$ in the Parkes survey known as PKSCAT90 with flux density larger than $0.25 \mathrm{Jy}$. A similar ratio is present in the 1 Jy-ARN survey. The space density of blazar in this survey is therefore 0.06 objects per square degree. We convert $2.7 \mathrm{GHz}$ fluxes to $5 \mathrm{GHz}$ and plot the density as an open diamond in Fig. 2.

We note that 84 additional sources are classified by Wall et al. (2004) as flat radio spectrum galaxies (57 of which without redshift); as some of these may well be BL Lacs, the blazar content of this sample will probably grow in the future.

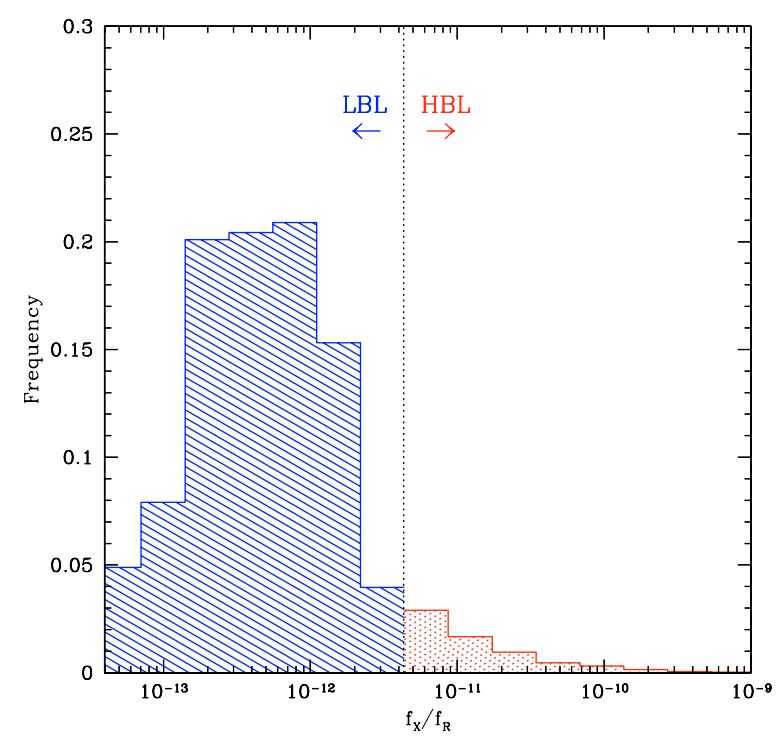

Fig. 4. The $f_{\mathrm{x}} / f_{\mathrm{r}}$ distribution of Blazars estimated from the $1 \mathrm{Jy}-\mathrm{ANR}$ and from a sample of about $2000 \mathrm{HBL}$ Blazar candidates (see text for details).

\subsection{The DXRBS blazar survey}

The Deep X-Ray Radio Blazar Survey (DXRBS) is a radio flux limited sample based on a double selection technique at radio and X-ray frequencies, which uses optical data to refine the sample. DXRBS searches for blazar candidates among serendipitous X-ray sources of the ROSAT PSPC pointed observations listed in the WGA catalog (White et al. 1995), restricting the sample to objects with radio spectral index flatter than $\alpha_{\mathrm{r}}=0.5$ and with broad-band spectral indices $\alpha_{\text {ro }}$ and $\alpha_{\mathrm{ox}}$ in the region occupied by blazars. Details of the selection method and of the optical identification of the candidates are described in Perlman et al. (1998), Landt et al. (2001), and Padovani et al. (2005).

Although the radio limit of the survey is $50 \mathrm{mJy}$ at $5 \mathrm{GHz}$, the X-ray sensitivity of the ROSAT PSPC pointings is not deep enough to ensure completeness at all radio fluxes, as some blazars with flux lower than $100 \mathrm{mJy}$ are expected to have an X-ray flux below the ROSAT WGA limit of $\approx 2 \times$ $10^{-14} \mathrm{erg}^{-2} \mathrm{~cm}^{-1}$. We therefore estimated the blazar space density the latest $\log N-\log S$ results of Padovani et al. (2005) taking into account for both FSRQs and BL Lacs and correcting the points at 50 and $100 \mathrm{mJy}$ for the fraction of lost objects as predicted from the distribution of Fig. 4. The final blazar space density from this survey is plotted in Fig. 2 as open triangles.

\subsection{The ASDC-XMM-Newton-NVSS (AXN) Sample}

In order to push the radio limit to fluxes significantly below $50 \mathrm{mJy}$ at $5 \mathrm{GHz}$ using the same multi-frequency selection method of the DXRBS survey, it is necessary to reach X-ray sensitivities proportionately deeper than that of the ROSAT-PSPC. To this purpose, we searched for serendipitous NVSS radio sources in XMM-Newton EPIC-pn (Strüder et al. 2001) X-ray images, which provide approximately one order of magnitude better sensitivity than ROSAT. 
Table 3. NVSS radio sources detected in XMM-Newton EPIC-pn $\mathrm{X}$-ray images.

\begin{tabular}{cccc}
\hline \hline $\begin{array}{c}\text { Number } \\
\text { of sources }\end{array}$ & $\begin{array}{c}\text { Space density } \\
\text { sources deg }^{-1}\end{array}$ & $\begin{array}{c}\text { Flux limit } \\
1.4 \mathrm{GHz}\end{array}$ & $\begin{array}{c}\text { Flux limit } \\
5 \mathrm{GHz}^{*}\end{array}$ \\
\hline 50 & $1.9 \pm 0.3$ & 50 & 31 \\
107 & $>4.0$ & 20 & 12 \\
149 & $>5.6$ & 10 & 6 \\
\hline
\end{tabular}

${ }^{*}$ We assume a spectral slope $\alpha_{\mathrm{r}}=0.4$.

We used the data in the XMM-Newton public archive available at the ASI Science Data Center (ASDC) in December 2004. At that date a standard processing, which removes periods of high background resulting from solar flares and detects serendipitous sources, had already been run at ASDC on 1220 EPIC-pn fields, 847 of which at high galactic latitude $(|b|>20)$.

Of these EPIC-pn observations, we considered the subsample of 188 non-overlapping fields that satisfy the conditions listed below that are necessary to ensure that the sample of serendipitous sources is suitable for our purposes:

1. the EPIC-pn instrument was used in full window mode;

2. the pointing of the XMM observation was at declination north of -40 degrees to ensure overlap with NVSS survey;

3. no bright or extended source is present in the field of view;

4. whenever more than one exposure of the same field was available in the archive, the deeper one was taken.

In addition, we have excluded a 5 arcmin circular area around the target of each field. The total area covered by the 188 fields is 26.3 square degrees. A careful inspection of all the $\mathrm{X}$ ray/radio/optical associations with broad-band spectral indices $\alpha_{\text {ro }}$ and $\alpha_{\text {ox }}$ in the region occupied by blazars (Giommi et al. 1999) led to the results summarized in Table 3.

For this first (conservative) estimation we assumed that all XMM fields are sufficiently deep to detect all blazars above $50 \mathrm{mJy}$. Because of the spread in exposure times and of the reduction in sensitivity at large off-axis angles due to vignetting effects and PSF degradation, approximately $20-30 \%$ of the objects might have been missed and the number of blazars found is only a lower limit. On the other hand, from the $1 \mathrm{Jy}-\mathrm{ARN}$ and other surveys, we expect that a similar percentage of candidates be associated to steep spectrum objects, so that the two effects roughly cancel out. We discuss the impact of this reduction in sensitivity in a future paper (Cavazzuti et al. 2005), where the sky coverage of the sample will be fully taken into account.

The space density at $50 \mathrm{mJy}$ at $1.4 \mathrm{GHz}(31 \mathrm{mJy}$ at $5 . \mathrm{GHz})$ has been plotted in Fig. 2 as a filled circle. The points at 12 and $6 \mathrm{mJy}$ are drawn as lower limits because in this case a significant fraction (e.g. $\gtrsim 50 \%$ ) of these faint blazars (especially at $6 \mathrm{mJy}$ ) is expected to be below the sensitivity limit of our XMM-Newton X-ray images. In fact, the $f_{\mathrm{x}} / f_{\mathrm{r}}$ distribution of known blazars (see Fig. 4) implies that the expected soft X-ray flux of a 10 mJy blazar is below $2 \times 10^{-15} \mathrm{erg} \mathrm{cm}^{-2} \mathrm{~s}^{-1}$ in about $30 \%$ of the cases. These X-ray sources could not be detected in most of our EPIC images, especially at large off-axis angles where most of the area is located.

\subsection{The Sedentary Survey of extreme high energy peaked BL Lacs}

We conclude this section summarizing some of the results from the multi-frequency "Sedentary Survey" (Giommi et al. 1999; Piranomonte et al. 2004; Giommi et al. 2005), which is a deep $\left(f_{\mathrm{r}} \geq 3.5 \mathrm{mJy}\right.$ at $\left.1.4 \mathrm{GHz}\right), 100 \%$ identified radio flux limited sample of 150 extreme HBL objects characterized by a $f_{\mathrm{x}} / f_{\mathrm{r}}$ ratio higher than $3 \times 10^{-10} \mathrm{erg} \mathrm{cm}^{-2} \mathrm{~s}^{-1} \mathrm{Jy}^{-1}$. This survey does not have a direct impact on the full blazar $\log N-\log S$ of Fig. 2, since such extreme HBL objects only represent a tiny minority of the full blazar population at radio frequencies. However, the very high $f_{\mathrm{x}} / f_{\mathrm{r}}$ flux ratio, which in these sources ranges from five hundred to over five thousand times that of typical blazars, makes these rare radio emitters a numerically important population of sources at $\mathrm{X}$-ray and $\mathrm{TeV}$ frequencies and therefore makes them potentially significant contributors to the Cosmic Background radiation at these frequencies. The space density of the objects at fluxes $f_{\mathrm{r}} \geq 6 \mathrm{mJy}(5 \mathrm{GHz})$ in this survey is reported in Fig. 2 as filled squares.

\section{From radio frequencies to other spectral bands}

Once the $\log N-\log S$ of a population of sources is known in a given energy band, it is possible to estimate their emission in other parts of the electromagnetic spectrum, provided that the flux ratio in the two bands, or even better, the overall energy distribution, is known. In this section we deal with flux ratios and SEDs of blazars that are used later in the paper to estimate the contribution to cosmic backgrounds at higher frequencies than radio.

The collection of SED of a large sample of blazars built using BeppoSAXand multi-frequency data presented by Giommi et al. (2002a) clearly shows that a wide variety of broad-band spectral forms exists. This large spectral diversity, however, can be reproduced, at least in a first approximation, by Synchrotron Self Compton (SSC) emission models such as those shown in Fig. 1. Sometimes, to explain the $\gamma$-ray part ofirstf the distribution, it is necessary to add other components in this simple picture. We will deal with this possibility later when we consider the contribution of blazars to the $\gamma$-ray background.

Although a continuous range of synchrotron peak energies probably exists, it is useful to divide the population of blazars into LBL whose X-ray emission is due to inverse Compton radiation, and in HBL characterized by a much higher X-ray flux (for the same radio flux), since their synchrotron component extends into the X-ray band. This simple separation into two broad categories also reflects the early selection methods, since LBL objects have mostly been discovered in radio surveys, while HBL objects have been typically found in X-ray flux limited samples. This large difference in X-ray emission in LBL and HBL objects is graphically reflected in the distribution of $f_{\mathrm{x}} / f_{\mathrm{r}}$ flux ratios shown in Fig. 4, which spans about 4 orders of magnitudes.

The plot shown in Fig. 4 was built using two radio flux limited samples. At $f_{\mathrm{x}} / f_{\mathrm{r}}$ values lower than $5 \times$ $10^{-12} \mathrm{erg} \mathrm{cm}^{-2} \mathrm{~s}^{-1} \mathrm{Jy}^{-1}$ (corresponding to LBL objects), we used the 1 Jy-ARN sample corrected for the RASS sky 
coverage. As the percentage of blazars sharply drops at $f_{\mathrm{x}} / f_{\mathrm{r}}$ values larger than $5 \times 10^{-12}$ erg $\mathrm{cm}^{-2} \mathrm{~s}^{-1} \mathrm{Jy}^{-1}$, the $1 \mathrm{Jy}-$ ARN sample rapidly becomes statistically inadequate and lager samples are clearly needed. We have therefore built a radio flux limited sample including nearly 2000 blazar candidates with $f_{\mathrm{x}} / f_{\mathrm{r}}>5 \times 10^{-12} \mathrm{erg} \mathrm{cm}^{-2} \mathrm{~s}^{-1} \mathrm{Jy}^{-1}$ and applying the same technique as used for the Sedentary survey, which is about $85 \%$ efficient in selecting HBL blazars with $f_{\mathrm{x}} / f_{\mathrm{r}}>$ $3 \times 10^{-10} \mathrm{erg} \mathrm{cm}^{-2} \mathrm{~s}^{-1} \mathrm{Jy}^{-1}$ (Giommi et al. 2005).

To that purpose, we cross-correlated the positions of the X-ray sources in the ROSAT All Sky Survey (Voges et al. 1999) with the radio sources of the NVSS catalog (Condon et al. 1998 ) and we estimated optical ( $J$ or $F$ ) magnitudes from the GSC2 Guide Star Catalog (McLean et al. 2000). We then calculated the broad-band $\alpha_{\text {ox }}$ and $\alpha_{\text {ro }}$ spectral slopes and only accepted objects in the area of the $\alpha_{\text {ox }}-\alpha_{\text {ro }}$ plane occupied by blazars (Giommi et al. 1999). Whenever no optical counterpart was found within the radio positional uncertainty, we assumed a lower limit of $J$ mag $=19.5$, which is the limit of the GSC2 catalog.

To estimate the distribution at $f_{\mathrm{x}} / f_{\mathrm{r}}>5 \times$ $10^{-12} \mathrm{erg} \mathrm{cm}^{-2} \mathrm{~s}^{-1} \mathrm{Jy}^{-1}$, the radio flux limit of the sample must correspond to an X-ray flux at which the ROSAT survey covers a large enough sky area to allow the detection of at least a few objects. We chose $f_{\mathrm{r}}=25 \mathrm{mJy}$, corresponding to $f_{\mathrm{X}}>1.25 \times 10^{-13} \mathrm{erg} \mathrm{cm}^{-2} \mathrm{~s}^{-1}$ where the RASS covers about 2000 square degrees of sky. We have then derived the tail of the $f_{\mathrm{x}} / f_{\mathrm{r}}$ distribution for $f_{\mathrm{x}} / f_{\mathrm{r}}<5 \times 10^{-12} \mathrm{erg} \mathrm{cm}^{-2} \mathrm{~s}^{-1} \mathrm{Jy}^{-1}$, taking the RASS sky coverage shown in Fig. 3 into account.

We checked the reliability of this selection method using the subsample of 514 objects for which Sloan Digital Sky Survey (SDSS) (e.g., York et al. 2000; Stoughton et al. 2002) spectral data (Turriziani et al. 2005) are available and we found that over $80 \%$ of the 319 candidates with $f_{\mathrm{x}} / f_{\mathrm{r}}>$ $5 \times 10^{-12} \mathrm{erg} \mathrm{cm}^{-2} \mathrm{~s}^{-1} \mathrm{Jy}^{-1}$ are indeed spectroscopically confirmed blazars. To properly normalize this distribution, we scaled it so that the fraction of blazars with $f_{\mathrm{x}} / f_{\mathrm{r}}>3 \times$ $10^{-10} \mathrm{erg} \mathrm{cm}^{-2} \mathrm{~s}^{-1} \mathrm{Jy}^{-1}$ (the $f_{\mathrm{x}} / f_{\mathrm{r}}$ limit of the Sedentary survey) is equal to the density ratio between the full blazar population and that of the extreme HBL of the sedentary survey (dashed line and filled squares in Fig. 2).

The combined $f_{\mathrm{x}} / f_{\mathrm{r}}$ distribution is shown in Fig. 4 where we see that the large majority $(94 \%)$ of blazars are of the LBL type, defined here as objects with $f_{\mathrm{x}} / f_{\mathrm{r}}<5 \times$ $10^{-12} \mathrm{erg} \mathrm{cm}^{-2} \mathrm{~s}^{-1} \mathrm{Jy}^{-1}$.

\section{The contribution of blazars to cosmic backgrounds}

Figure 5 shows the spectral energy distribution of the cosmic extragalactic background radiation in the microwave, X-ray and $\gamma$-ray band, that is where we expect that the blazar collective emission gives a significant contribution.

The CMB, taken from the very accurate measurements of the COBE satellite is represented by a black-body spectrum with temperature of $2.725^{\circ} \mathrm{K}$ (Mather et al. 1999); the CXB is taken from HEAO-1 measurements (Marshall et al. 1980; Gruber et al. 1999) and has been scaled to match the more

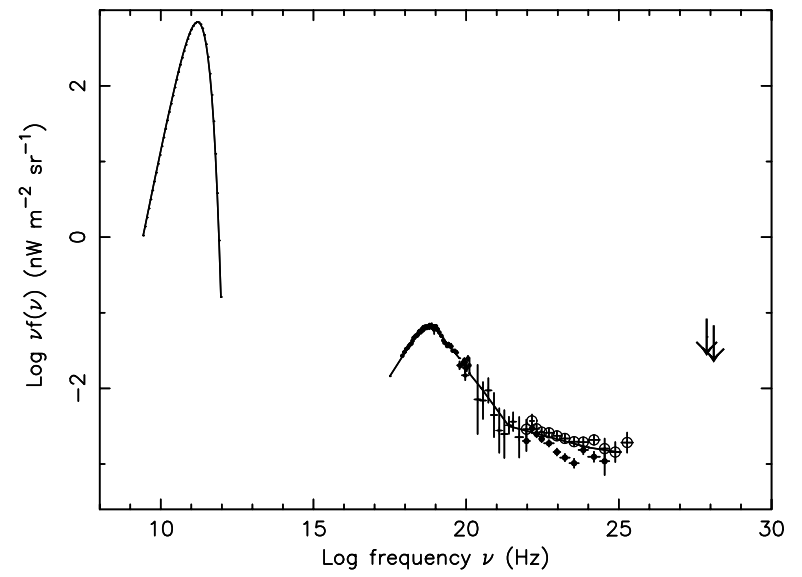

Fig. 5. The extragalactic Cosmic Background Energy distribution at microwave, $\mathrm{X}$-ray and $\gamma$-ray energies.

recent BeppoSAX, ASCA, and XMM-Newton results in the softer 2-10 keV band (Vecchi et al. 1999; Lumb et al. 2002; Kushino et al. 2002). The gamma ray background is derived from the COMPTEL data in the range $0.8-30 \mathrm{MeV}$ (Kappadath 1998) and from EGRET data in the range $30 \mathrm{MeV}-50 \mathrm{GeV}$. We recall that the estimate of the extragalactic $\gamma$-ray background emission depends on the Galactic diffuse emission model, which itself is not yet firmly established. In Fig. 5 we report the results of two different analyses of the EGRET data: open circles from Sreekumar et al. (1998) and filled circles from Strong et al. (2004), which uses an improved model of the Galactic diffuse continuum gamma-rays. As for the TeV diffuse background, we report the upper limits in the $20-100 \mathrm{TeV}$ region derived from the HEGRA air shower data analysis (Aharonian et al. 2002). Note that the HEGRA measurements are sensitive to both non-isotropic (galactic) and isotropic (extragalactic) component. In the $1 \mathrm{TeV}-1 \mathrm{PeV}$ energy range, other experiments give only upper limits and there is no clear observation of a diffuse photon signal yet.

In the following we estimate the blazar contribution to the cosmic backgrounds described above basing our calculation on the radio $\log N-\log S$ built in Sect. 2 and on flux ratios in different bands or on the observed Spectral Energy Distributions of $\gamma$-ray detected sources.

\subsection{The Cosmic Microwave Background}

The contribution of blazars to the CMB has been estimated in the past from different viewpoints (see, e.g. Toffolatti et al. 1998; Giommi \& Colafrancesco 2004). Here we use the blazar radio $\log N-\log S$ of Fig. 2 to update the results presented in Giommi \& Colafrancesco (2004).

From Fig. 2 we see that the blazar $\log N-\log S$ can be represented by a broken power law model with alpha $=1.62$ (integral slope) up to a break point where the slope flattens significantly. The precise position of the break and the amount of flattening cannot be estimated with our data. However, a break must occur somewhere around 10-15 mJy, otherwise the number of blazars would exceed the total number of radio sources at about 2-3 mJy. In the following we use the somewhat 


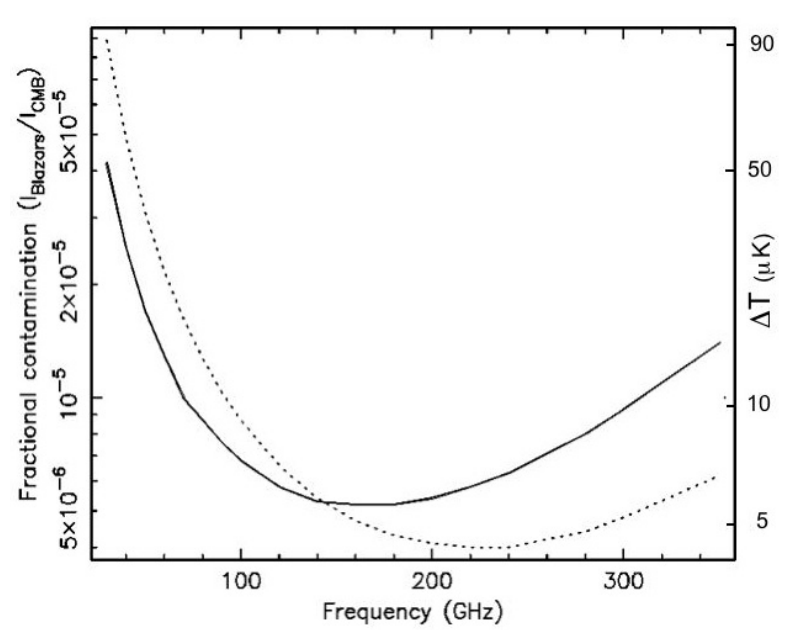

Fig. 6. The Blazar fractional contamination of the CMB (solid line) and the apparent increase in CMB temperature due to Blazars (dotted line) as a function of frequency.

conservative values of $15 \mathrm{mJy}$ for the break and 0.9 for the slope below the break. Both values are consistent with the lower limits on the blazar counts at 12 and 6 mJy (see Sect. 2)

The integrated background intensity due to blazars therefore can be expressed as

$I_{\text {blazars }}=\int_{0.1 \mathrm{mJy}}^{1 \mathrm{Jy}} S \frac{\mathrm{d} N}{\mathrm{~d} S} \mathrm{~d} S$,

where $\mathrm{d} N / \mathrm{d} S$ is the differential of Eq. (1). The minimum integration flux of $0.1 \mathrm{mJy}$ for $S_{\min }$ is likely to be conservative, since blazars with radio flux near or below $1 \mathrm{mJy}$ are already included in the Einstein Medium Sensitivity Survey BL Lac sample (Rector et al. 2000). The integrated intensity $I_{\text {blazars }}$ is then converted from $5 \mathrm{GHz}$ to microwave frequencies by convolving the flux value with the observed distribution of spectral slopes between $5 \mathrm{GHz}$ to microwave frequencies, as estimated from the 1 Jy-ARN sample (see Giommi \& Colafrancesco 2004, for details).

Figure 6 plots the fractional contamination, defined as $I_{\text {blazars }} / I_{\mathrm{CMB}}$ : that is, the ratio between the blazar integrated emission and the CMB intensity (solid line) and the equivalent apparent temperature increase of the CMB (dotted line), as a function of frequency.

The contribution of blazars to the temperature anisotropy spectrum of the $\mathrm{CMB}$ is calculated as $(\Delta T)_{\text {blazar }}=$ $\left[(2 \pi)^{-1} C_{\ell} \ell(\ell+1)\right]^{1 / 2}$, where

$C_{\ell, \text { blazar }}=\int_{S_{\text {min }}}^{S_{\max }} \mathrm{d} S \frac{\mathrm{d} N}{\mathrm{~d} S} S^{2}$,

assuming that the blazars are spatially distributed like a Poissonian random sample (Tegmark \& Efstathiou 1996; Scott \& White 1999; Giommi \& Colafrancesco 2004). The quantity on the right hand side in Eq. (3) is the usual Poisson shot-noise term (Peebles 1980; Tegmark \& Efstathiou 1996). We neglect here the clustering term, $\omega(I)^{2}$, which adds to the Poissonian term since there is not a clear estimate of the blazar clustering yet. We note, however, that the inclusion of clustering can significantly increase the amount of contamination, especially at large angular scales (Gonzales-Nuevo et al. 2005).
For the blazar population described by the $\log N-\log S$ given in Fig. 2, we found $C_{\ell \text {,blazar }} \approx 54.4 \mathrm{Jy}^{2} \mathrm{sr}^{-1}$ at $41 \mathrm{GHz}$ and $C_{\ell \text {,blazar }} \approx 51.6 \mathrm{Jy}^{2} \mathrm{sr}^{-1}$ at $94 \mathrm{GHz}$. Our results can be translated into temperature units using the conversion between the isotropic black-body (Planckian) brightness $B_{0}(v)$ and the CMB temperature $T_{0}$, which can be written as

$$
\begin{aligned}
\frac{\partial B_{0}}{\partial T_{0}} & =\frac{k}{2}\left(\frac{k T_{0}}{h c}\right)^{2} \times\left[\frac{x^{2}}{\sinh (x / 2)}\right]^{2} \\
& =\left(\frac{24.8 \mathrm{Jy} \mathrm{sr}^{-1}}{\mu \mathrm{K}}\right) \times\left[\frac{x^{2}}{\sinh (x / 2)}\right]^{2}
\end{aligned}
$$

Here $x \equiv h v / k T_{0}=v / 56.84 \mathrm{GHz}$ is the a-dimensional frequency given in terms of the Planck constant $h$, of the speed of light $c$ and of the Boltzmann constant $k$. We found a value $C_{\ell \text {,blazar }} \approx 2.22 \times 10^{-2} \mu \mathrm{K}^{2}$ sr at $41 \mathrm{GHz}$ and $C_{\ell \text {,blazar }} \approx 1.09 \times$ $10^{-3} \mu \mathrm{K}^{2}$ sr at $94 \mathrm{GHz}$. We show in Figs. 7 and 8 the quantity $\Delta T_{\text {blazar }}$ and compare it to the CMB fluctuation power spectrum which best fits the available data. The previous value is shown as solid lines and has to be considered as a definite lower limit for $C_{\ell \text {,blazar }}$, since it neglects the contribution of steep-spectrum sources at low frequencies which flatten at these frequencies (41 and $94 \mathrm{GHz}$ ) and the effect of flux variability. The dashed lines in Figs. 7 and 8 show the blazar power spectrum by adding the contribution of radio sources with steep-spectrum at low radio frequencies, which flatten at higher frequencies. The effect of spectral and flux variability also allows for an increase in the fluctuation level $C_{\ell \text {,blazar }}$, because many blazars below the sensitivity threshold of CMB experiments can be detected during flares. The blazar flux variability at millimeter wavelengths may be very substantial (higher than factors $3-10$ on time scales of weeks to years seen at $\mathrm{cm}$ wavelengths) and could definitely increase the level of contamination of CMB maps when these are built over long integration periods. The dotted lines in Figs. 7 and 8 show the blazar power spectrum, when this last contribution is also taken into account.

Our numerical estimates are subject to some uncertainties that depend on the parameters of the $\log N-\log S$ that are not constrained well. For instance, if instead of using the conservative values of $15 \mathrm{mJy}$ and 0.9 for the break flux and slope below the break, we used the less conservative values of $10 \mathrm{mJy}$ and $\alpha=1.0$, we would have an increase of $\approx 50 \%$ for $I_{\text {blazars }}$ and of $\approx 4 \%$ for $C_{\ell \text {,blazar }}$.

The contamination level shown in Figs. 7 and 8 is the one expected in the case where no blazars are removed from the CMB data. The observed WMAP angular power spectrum reported by Hinshaw (2003) was obtained using a procedure in which a pure primordial signal was initially assumed, and then subtracting the foreground sources that could be recognized as such in the WMAP data, thereby removing only the bright tail of the blazar $\log N-\log S$ distribution. The procedure adopted to derive the $\mathrm{CMB}$ power spectrum should take into account the full point-like source contribution implied by our $\log N-\log S$. Such an approach would both influence the shape of the expected power spectrum and increase the statistical uncertainties of the WMAP data points, especially at high multipoles, where the blazar contribution is larger. 


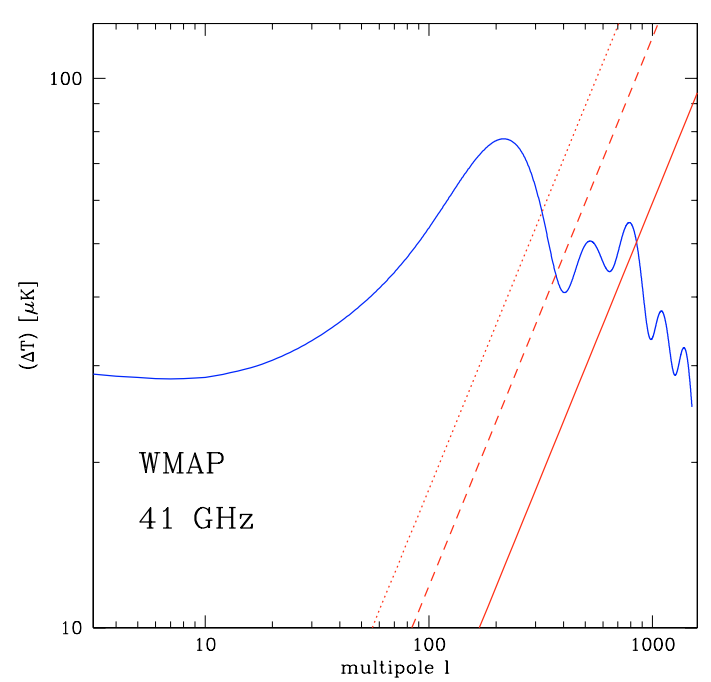

Fig. 7. The contribution of Blazars to the CMB fluctuation spectrum in the WMAP Q channel at $41 \mathrm{GHz}$ as evaluated from the $\log N-\log S$ given in Fig. 2 (solid line). We also show the angular power spectrum for the Blazar population by adding an estimate of the possible contribution of radio sources with steep-spectrum at low radio frequencies which flatten at higher frequencies (dashed line). The dotted line includes also the effect of spectral and flux variability (see text for details). Although this additional contamination may be substantial a precise estimation can only be done through simultaneous high resolution observations at the same frequency. A typical CMB power spectrum evaluated in a $\Lambda$ CDM cosmology with $\Omega_{\mathrm{m}}=0.3, \Omega_{\Lambda}=$ $0.65, \Omega_{b}=0.05$ which best fits the available data is shown for comparison.

We stress that the previous calculations we performed neglecting the clustering term $\omega(I)^{2}$ and thus they must again be considered as a lower limit to the realistic angular power spectrum contributed by the blazars. The effects of the clustering of FSRQ on the CMB fluctuation spectrum has been partially estimated by some authors: for instance Gonzales-Nuevo et al. (2005) used simulations to calculate that the clustering of extragalactic radio sources is rather small at $\ell=200$ for the Planck frequencies, while the contribution of the clustering term to the confusion noise is likely to be the dominant one. Scott \& White (1999) estimate instead that the clustering of SCUBA sources to the expected Planck CMB spectrum leads to an increase of the contamination from point-like sources of a factor 5-10 with respect to the Poissonian term at $\ell=200$. Expectations for the clustering effect strongly depend both on the adopted model for the source counts and on their clustering model. Based on the correlation function of Loan et al. (1997) (adopted by Toffolatti), on that for the SCUBA sources (adopted by Scott $\&$ White 1999), and on our blazar $\log N-\log S$, we expect that the contamination of the first peak of the fluctuation spectrum (at the WMAP $41 \mathrm{GHz}$ channel) is at a level in the range $20-25 \%$. This estimate does not include possible variability effects and additional core-dominated radio sources

\subsection{The soft $X$-ray background}

We estimated the contribution of blazars to the CXB at $1 \mathrm{keV}$ using two methods: i) converting the integrated radio flux

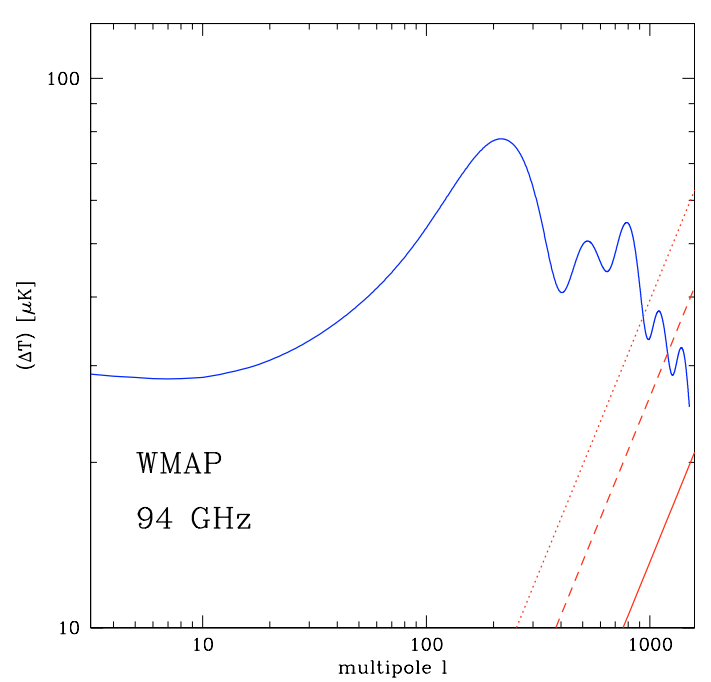

Fig. 8. The contribution of Blazars to the CMB fluctuation spectrum as in Fig. 7 for the WMAP $94 \mathrm{GHz}$ channel.

calculated with Eq. (2) into X-ray flux with the observed distribution of $f_{\mathrm{x}} / f_{\mathrm{r}}$ flux ratios in Fig. 4; and ii) converting the integrated blazar contribution to the CMB (at $94 \mathrm{GHz}$ ) to Xray flux using the distribution of $\alpha_{\mu x}$, the microwave $(94 \mathrm{GHz}$ ) to X-ray (1 keV) spectral slope, defined as

$\alpha_{\mu x}=-\frac{\log \left(f_{1 \mathrm{keV}} / f_{94 \mathrm{GHz}}\right)}{\log \left(v_{1 \mathrm{keV}} / v_{94 \mathrm{GHz}}\right)}=-\frac{\log \left(f_{1 \mathrm{keV}} / f_{94 \mathrm{GHz}}\right)}{6.41}$,

and estimated from the subsample of blazars detected by WMAP in the $94 \mathrm{GHz}$ channel, for which an X-ray measurement is available (see Fig. 9).

The first method gives a total blazar contribution to the X-ray background of $2.7 \times 10^{-12} \mathrm{erg} \mathrm{cm}^{-2} \mathrm{~s}^{-1} \mathrm{deg}^{-1}$ (about $70 \%$ of which is due to HBL sources with $f_{\mathrm{x}} / f_{\mathrm{r}}>$ $5 \times 10^{-12} \mathrm{erg} \mathrm{cm}^{-2} \mathrm{~s}^{-1} \mathrm{Jy}^{-1}$ ) in the ROSAT $0.1-2.4 \mathrm{keV}$ energy band. Assuming an average blazar X-ray energy spectral index of $\alpha_{\mathrm{x}}=0.7$, which is a compromise between a flatter spectrum that is typical of LBL sources and an $\alpha_{\mathrm{x}}$ steeper than 1 for HBL objects, this flux converts to $2.6 \times 10^{-12} \mathrm{erg} \mathrm{cm}^{-2} \mathrm{~s}^{-1} \mathrm{deg}^{-1}$ in the $2-10 \mathrm{keV}$ band or $11 \%$ of the $\mathrm{CXB}$, which is estimated to be $2.3 \times 10^{-11} \mathrm{erg} \mathrm{cm}^{-2} \mathrm{~s}^{-1} \mathrm{deg}^{-1}$ (Perri \& Giommi 2000).

The distribution of microwave to X-ray spectral slope in Fig. 9 has an average value of $\left\langle\alpha_{\mu x}\right\rangle=1.07$ and a standard deviation of 0.08 corresponding only to about a factor 3 in flux. This distribution is much narrower than the one between the radio and X-ray band (Fig. $4, f_{\mathrm{x}} / f_{\mathrm{r}}$ ), while the dispersion is comparable to the variability that blazars show at radio and especially at X-ray frequencies.

Since the $94 \mathrm{GHz}$ and X-ray measurements are not simultaneous, most of the dispersion in the distribution shown in Fig. 9 is probably due to variability both in the microwave and X-ray bands. The intrinsic dispersion is therefore likely to be much smaller. For this reason, in converting from the background level at $94 \mathrm{GHz}$ estimated in the previous section to $1 \mathrm{keV}$, we used the average $\alpha_{\mu x}$ value and assumed no dispersion. We must note, however, that just like the 1 Jy radio surveys, the WMAP $\alpha_{\mu x}$ distribution hardly includes any HBL sources, which make up only a few percent of the population. 


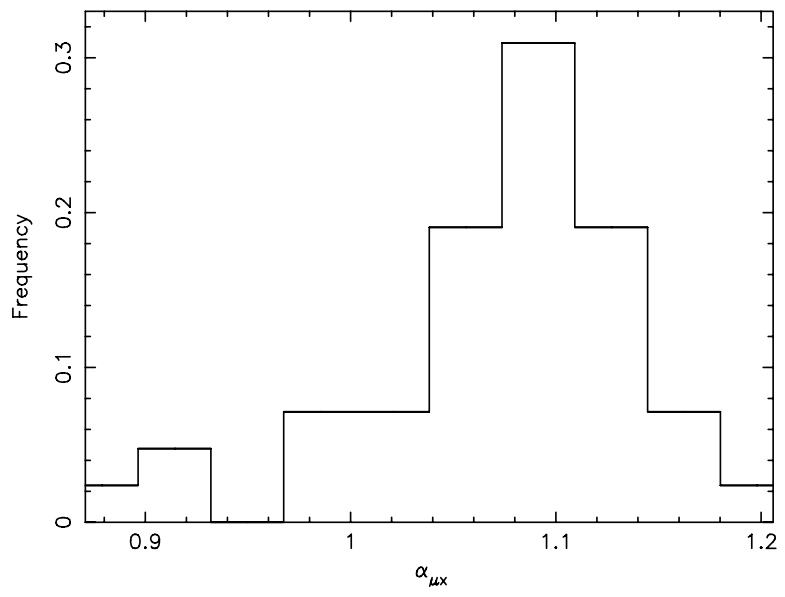

Fig. 9. The distribution of spectral slopes between the microwave $(94 \mathrm{GHz})$ and X-ray $(1 \mathrm{keV})$ band measured from the sample of 42 Blazars included in the WMAP catalog of bright microwave sources detected in the $94 \mathrm{GHz}$ channel and for which X-ray measurements are available.

From Eq. (5) we get

$f_{1 \mathrm{keV}}=1.4 \times 10^{-7} f_{94 \mathrm{GHz}}$.

Since the blazar integrated emission at $94 \mathrm{GHz}$ is $7.2 \times$ $10^{-6} \mathrm{CMB}_{94 \mathrm{GHz}}$ or $0.64 \mathrm{Jy} / \mathrm{deg}^{2}$ and the cosmic X-ray background is $2.3 \times 10^{-11} \mathrm{erg} \mathrm{cm}^{-2} \mathrm{~s}^{-1}$ (Perri \& Giommi 2000) in the $2-10 \mathrm{keV}$ band, equivalent to $2.31 \mu \mathrm{Jy} / \mathrm{deg}^{2}$ at $1 \mathrm{keV}$, Eq. (6) gives $f_{1 \mathrm{keV}}=0.09 \mu \mathrm{Jy} / \mathrm{deg}^{2}$ or $3.9 \%$ of the CXB for $f_{94 \mathrm{GHz}}=0.64 \mathrm{Jy} / \mathrm{deg}^{2}$.

Considering that the distribution of Fig. 9 only includes LBL objects and that HBL sources make up two thirds the total contribution to the CXB (see above), the percentage scales to about $12 \%$ which is very close to the $11 \%$ obtained with the previous method. Both results are in good agreement with the independent estimate based on the XMM-Newton Bright Serendipitous Survey by Galbiati et al. (2005), who conclude that the radio loud AGN content of the CXB is $13 \%$.

\subsection{Hard X-ray - soft $\gamma$-ray background}

The number of sources detected at higher energies than soft $\mathrm{X}$-rays is still rather low, so building reliable distributions of flux ratios between radio or microwaves and the Hard $\mathrm{X}$-ray $/ \gamma$-ray fluxes similar to those of Figs. 4 and 9 is not currently possible. In order to estimate the blazar contribution to high energy Cosmic Backgrounds ( $E>100 \mathrm{keV})$, we therefore followed a different approach. We extrapolated the predicted blazar integrated intensity at microwave frequencies (Eq. (2)) to the hard X-ray and soft $\gamma$-ray band using a set of hypothetical SSC spectral energy distributions.

Figure 10 shows the $\mathrm{CMB}, \mathrm{CXB}$, and CGB together with three predicted SED from a simple homogeneous SSC models whose parameters are constrained to 1) be consistent with the expected integrated flux at $94 \mathrm{GHz} ; 2$ ) have the $\alpha_{\mu x}$ slope equal to the mean value of the WMAP blazars $\left(\alpha_{\mu x}=1.07\right)$; and 3 ) possess a radio spectral slope equal to the average value in the WMAP sample. The three curves, forced to pass through

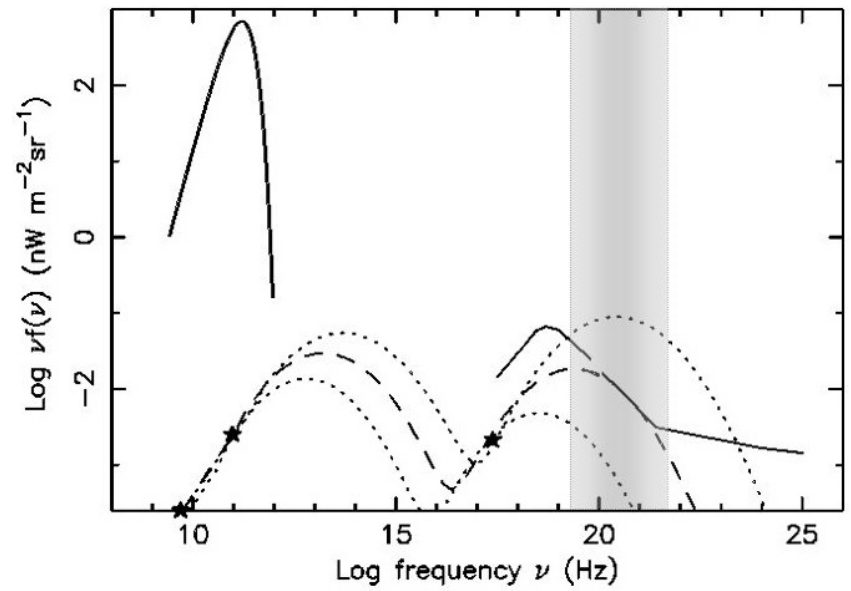

Fig. 10. The possible contribution of LBL Blazars to the Hard $\mathrm{X}$-ray/soft $\gamma$-ray Background (shaded area). The three SSC curves corresponds to different $v_{\text {peak }}$ values $\left(\log v_{\text {peak }}=12.8,13.5\right.$ and 13.8) and are constrained to go through the three star symbols representing 1$)$ the total contribution of Blazars at $94 \mathrm{GHz}\left(8 \times 10^{-6}\right.$ of the CMB intensity), 2) the average $5 \mathrm{GHz}$ to $94 \mathrm{GHz}$ slope $\left(\alpha_{5-94 \mathrm{GHz}}=0.2\right)$ and 3$)$ the average spectral slope between $94 \mathrm{GHz}$ and $1 \mathrm{keV}\left(\left\langle\alpha_{\mu x}\right\rangle=1.07\right)$.

the three star-symbols graphically representing the three constraints listed above, are characterized by synchrotron peak frequencies of $v_{\text {peak }}=10^{12.8}, 10^{13.5}$, and $10^{13.8} \mathrm{~Hz}$. From Fig. 10 we see that a high value of $v_{\text {peak }}$ by a large factor overestimates the observed hard-X-ray to soft $\gamma$-ray $\left(\approx 10^{20}-2 \times 10^{22} \mathrm{~Hz}\right.$ or $\approx 500 \mathrm{keV}-10 \mathrm{MeV}$ ) cosmic background, whereas a too low value of $v_{\text {peak }}$ predicts a negligible contribution. The case $v_{\text {peak }}=10^{13.5} \mathrm{~Hz}$ predicts $100 \%$ of the cosmic background. Since the $\log \left(v_{\text {peak }}\right)$ values of blazars in the 1 Jy-ARN survey and WMAP catalog peak near 13.5 and range from 12.8-13.7 within one sigma from the mean value, we argue that the data presently available indicate that blazars may be responsible for a large fraction, possibly $100 \%$, of the hard-Xray/soft $\gamma$-ray cosmic background.

\section{4. $\gamma$-ray background}

The SSC distributions of Fig. 10 predict a negligible blazar contribution to the extragalactic $\gamma$-ray Background above $100 \mathrm{MeV}$. Nevertheless, it is well known that blazars are the large majority of the extragalactic $\gamma$-ray $(E>100 \mathrm{MeV})$ identified sources detected by the EGRET experiment (Hartman et al. 1999) aboard the Compton Gamma-ray Observatory; therefore they are likely to contribute to the $\gamma$-ray background in a significant way. Indeed, Padovani et al. (1993) concluded that blazars should make a large fraction, if not the totality, of the extragalactic $\gamma$-ray background on the basis of a small number of sources detected by EGRET. However, these early calculations relied upon a small database and had to assume no strong variability, a characteristic that was later demonstrated to be very common in $\gamma$-ray detected blazars.

Instead of considering simple average values of the radio to $\gamma$-ray flux ratio as in Padovani et al. (1993), we estimated the blazar contribution to the $\gamma$-ray background using the full SED of blazars, scaling it to the integrated blazar flux intensity 


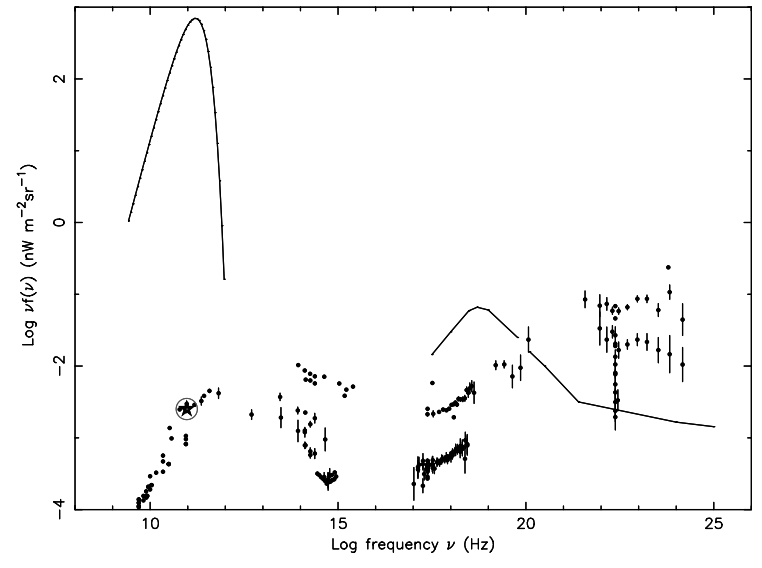

Fig. 11. The CMB, $X$-ray and $\gamma$-ray cosmic backgrounds with superimposed the SED of the Blazar 3C 279 scaled so that its flux at $94 \mathrm{GHz}$ matches the cumulative emission of the entire Blazar population (encircled star symbol).

as calculated with Eq. (2). Figure 11 compares the energy distribution of the CMB, CXB, and CGB to the SED of 3C 279, a well-known bright blazar detected by EGRET, scaled as explained above.

Figure 11 shows the large variability of 3C 279 in the X-ray and $\gamma$-ray band. While the contribution to the CXB ranges from a few percent to over $10 \%$ in the higher states, the predicted flux at $\gamma$-ray frequencies ranges from about $100 \%$ to several times the observed cosmic background intensity. This large excess implies that either 3C 279 is very non representative of the class of blazars, even though the contribution to the CXB is consistent with other estimates, or its duty cycle at $\gamma$-ray frequencies is very low (see Fig. 12). The same approach can be followed with other blazars detected at $\gamma$-ray frequencies. In most EGRET detected blazars the SED of LBL blazars overestimates the CGB by a large factor.

A way of quantifying the ratio between the $\gamma$-ray intensity predicted by assuming that the source is representative of the entire population and the actual background intensity is to use, in analogy with Eq. (5), a microwave (94 GHz) to $\gamma$-ray $(100 \mathrm{MeV})$ slope $\alpha_{\mu \gamma}$ :

$\alpha_{\mu \gamma}=-\frac{\log \left(f_{94 \mathrm{GHz}} / f_{100 \mathrm{MeV}}\right)}{\log \left(v_{94 \mathrm{GHz}} / v_{100 \mathrm{MeV}}\right)}=\frac{\log \left(f_{94 \mathrm{GHz}} / f_{100 \mathrm{MeV}}\right)}{11.41}$.

We also defined an $\alpha_{\mu \gamma_{100 \% \mathrm{CGB}}}=0.994$ as the $\alpha_{\mu \gamma}$ of a hypothetical source that would produce $100 \%$ of the CGB if representative of the entire class. This value is based on the CGB intensity at $100 \mathrm{MeV}$ shown in Fig. 5. Any real source with $\alpha_{\mu \gamma}$ flatter than $\alpha_{\mu \gamma_{100 \% \mathrm{CGB}}}=0.994$ would then predict an integrated flux in excess of the observed $\gamma$-ray background if representative of the entire population; alternatively, its duty cycle must be lower than $100 \%$.

Table 4 lists the main properties of the 34 blazars detected by WMAP and by EGRET. Column 1 gives the source name, Cols. 2 and 3 give the right ascension and declination, Col. 4 gives the $5 \mathrm{GHz}$ radio flux, Col. 5 gives the $94 \mathrm{GHz}$ microwave flux measured by WMAP or estimated from the extrapolation from measurements in lower WMAP channels or from the literature, Col. 6 gives the minimum and maximum $\gamma$-ray flux

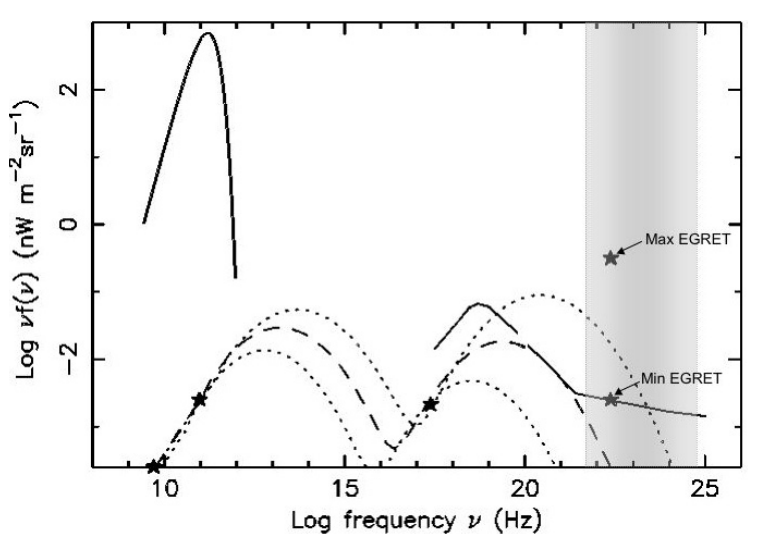

Fig. 12. The possible contribution of LBL Blazars to the $\gamma$-ray Cosmic Background (shaded area). Simple SSC energy distributions fail to reproduce the observed intensity and slope of the $\gamma$-ray Background. The observed range of $\gamma$-ray emission with EGRET, normalized to radio flux, indicates that the duty cycle at these energies must be low (see text for details).

measured by EGRET (excluding lower limits) taken from the third EGRET catalog (Hartman et al. 1999), Col. 7 gives the corresponding $\alpha_{\mu \gamma}$ values, Col. 8 gives the maximum duty cycle allowed assuming that the source is representative of the entire blazar population, Cols. 9 and 10 give the EGRET and WMAP source names. We note that all sources in the list must have a duty cycle lower than $100 \%$.

\subsection{TeV background}

All blazars detected so far at TeV energies are of the HBL type. This is readily interpreted within the SSC scenario, since only objects where the synchrotron radiation extends to near or within the X-ray band can produce a corresponding inverse Compton flux that reaches $\mathrm{TeV}$ energies, at least assuming a single scattering.

In the following we estimate the blazar contribution to the $\mathrm{TeV}$ background in a graphical way as in the previous paragraph for the case of the $\gamma$-ray Background but only considering the HBL component and not the entire blazar population. From a comparison of the normalization of the blazar radio $\log N-\log S$ with that of the Sedentary survey (Giommi et al. 1999, see also Fig. 2 which is representative of extreme HBLs), we estimate that these objects are about $0.1 \%$ of the blazar population.

In Fig. 13 we plot the SED of the well known TeV blazar MKN421 normalized at $94 \mathrm{GHz}$, so that the flux is scaled to $0.1 \%$ of the intensity produced by the entire population of blazars.

From this figure we see that, despite HBLs being a tiny minority, their integrated X-ray flux makes up a fair fraction of the $\mathrm{CXB}$ and that their $\mathrm{TeV}$ emission may produce a significant amount of extragalactic light, even considering that the real flux level should be lower than the one shown in Fig. 13 at $\mathrm{TeV}$ energies, since only the objects closer than $z \approx 0.2$ can be detected at these energies.

We note, however, that since extreme HBLs, such as those of the Sedentary survey, are very rare (one object in several 


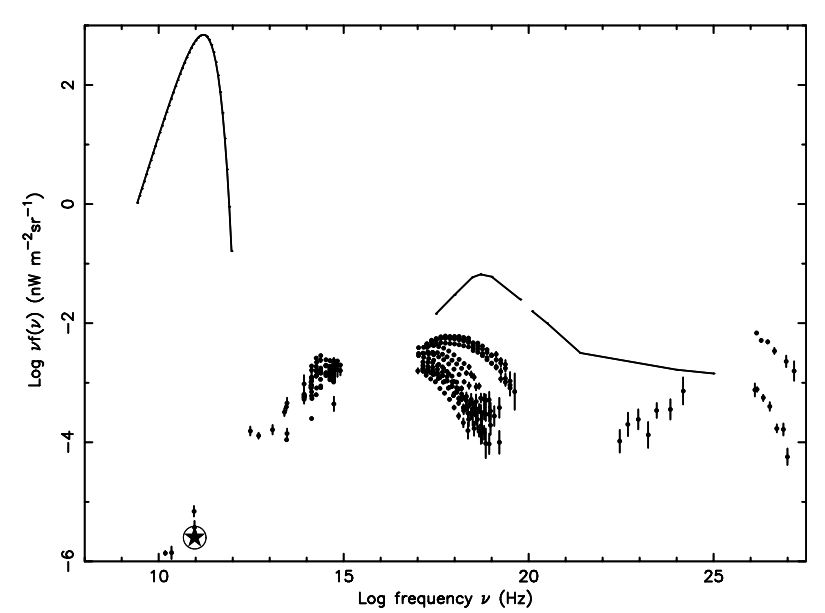

Fig. 13. The CMB, X-ray and $\gamma$-ray cosmic backgrounds with superimposed the SED of MKN 421 scaled to $1 / 1000$ of the total Blazar contribution to the CMB since the radio $\log N-\log S$ of extreme HBL like this source (in the sedentary survey) is about $1 / 1000$ of the $\log N-\log S$ of all Blazars.

thousand degrees with flux above a few mJy), the extragalactic light at $\mathrm{TeV}$ energies should be very patchy, associated to single sources, rather than a diffuse light resulting from the superposition of many unresolved discrete sources, as in the other cosmic backgrounds.

\section{Summary and discussion}

Blazars are the only type of AGN known to emit non-thermal radiation across the entire electromagnetic spectrum from radio waves to the most energetic $\gamma$-rays. In some energy bands, the power emitted by these sources is orders of magnitude larger than is generated through the accretion process by other types of AGN. At these frequencies blazars are the dominant population in the extragalactic sky despite their low space density and they contribute significantly to the extragalactic cosmic backgrounds.

A deep understanding of the blazar contribution to the cosmic background light is becoming an increasing necessity, as the microwave, the $\gamma$-ray and the $\mathrm{TeV}$ bands are about to be intensively explored by a new generation of astronomy satellites and ground-based Cherenkov telescopes.

The overall cosmic background energy has two well understood components: the primordial black body emission peaking at microwave frequencies, or $\mathrm{CMB}$, and the X-ray apparently diffuse emission arising from the accretion onto super-massive black holes in AGN integrated over cosmic time, or CXB. We have shown that blazars add a third non-thermal component that at low frequencies contaminates the CMB fluctuation spectrum and complicates its interpretation, while at the opposite end of the electromagnetic spectrum it dominates the extragalactic background radiation.

Our calculations are based on a new, deep blazar radio $\log N-\log S$ that was assembled by combining several radio and multi-frequency surveys. The integrated radio flux from the entire population was extrapolated to other energy bands using observed flux ratios and broad-band spectral energy distributions. Our results can be summarized as follows.

Contribution to the CMB. The results presented here confirm and extend the findings of Giommi \& Colafrancesco (2004). The blazar contribution to the cosmic background intensity at microwave frequencies ranges between $5 \times 10^{-5}$ to $5 \times 10^{-6}$ of the CMB, depending on observing wavelength. The consequences are twofold: $i)$ there is an apparent temperature excess of $5-50 \mu \mathrm{K}$, and ii) the CMB fluctuation spectrum is affected by a spurious signal that becomes significant or dominant at multipole $l \approx 300-600$ (see Figs. 7 and 8). Since blazars, like all cosmic sources, are probably not distributed in a completely random way across the sky, the source clustering may significantly increase the amount of contamination, particularly at large angular scales (Gonzales-Nuevo et al. 2005).

We also note that the temperature excess causes a bias in the statistical distribution of the primordial CMB fluctuation spectrum. If not properly removed, this non-thermal foreground radiation may really complicate the detection of primordial non-Gaussianity that may carry important information on the dynamics of the inflationary phase (e.g., Peebles 1997).

Contribution to the CXB. The contribution to the soft X-ray background has been derived by converting the integrated radio and microwave blazar emission into X-ray flux using observed flux ratio distributions. Our estimated values of $11-12 \%$ the CXB agree very well with the independent measurement of $13 \%$ for radio loud AGN obtained by Galbiati et al. (2005) using XMM-Newton data. For the most part, this X-ray flux is composed of emission due to the end of the synchrotron component of HBL objects, while for about one third the X-ray flux is due to the flat inverse Compton spectral component of LBL sources. Given the very different X-ray spectral slopes of LBL and HBL at higher energies than $1 \mathrm{keV}$, the blend between the (steep) synchrotron HBL component and the (flat) inverse Compton LBL component must change in favor of the latter. Since the spectral slope of the inverse Compton component is very similar to that of the CXB up to $40-50 \mathrm{keV}$, the LBL contribution should stay roughly constant around $4-5 \%$ up to that energy. Above $\approx 50 \mathrm{keV}$, the observed CXB steepens, and the contribution of LBL blazars should progressively increase. One of the methods that we used is based on the $f_{\mathrm{x}} / f_{\mathrm{r}}$ distribution of Fig. 4 where LBL sources are the large majority among blazars, while HBL are only about $5 \%$. We have converted the integrated radio flux into X-rays assuming that the $f_{\mathrm{x}} / f_{\mathrm{r}}$ distribution remains constant at all radio fluxes. A strong increase in the fraction of HBL at low radio luminosity (hence fluxes), as required by the blazar sequence within the unified schemes of Fossati et al. (1997) and Ghisellini et al. (1998), would result in a much larger blazar contribution to the CXB, inconsistent with observations. This conclusion, together with the findings of Giommi et al. (2002b), Padovani et al. (2003b), Caccianiga \& Marchã (2004), Giommi et al. (2005), and Nieppola et al. (2005) cast serious doubts on the validity of the blazar sequence. 
Contribution to the soft $\boldsymbol{\gamma}$-ray Background. At energies higher than $\approx 100 \mathrm{keV}$ the contribution to the Cosmic Background flux was estimated converting the expected blazar contribution to the diffuse background at microwave frequencies using SEDs predicted by simple homogeneous SSC models, constrained to have a microwave-to-X-ray spectral slope $\alpha_{\mu x}$ equal to the observed average value of 1.07 .

The hard X-ray/soft $\gamma$-ray background is reproduced both in shape (spectral slope) and intensity assuming an average SSC distribution where the synchrotron component peaks at $10^{13.5} \mathrm{~Hz}$, well within the range of observed values. We conclude that the Cosmic Background between $\approx 0.5$ and $\approx 10 \mathrm{MeV}$ is consistent with being due to the tail of the Inverse Compton component of LBL objects (see Fig. 10).

Contribution to the $\boldsymbol{\gamma}$-ray Background. The SED of blazars detected by EGRET predict much more $\gamma$-ray Background than observed so they cannot be representative of the entire population, at least in a stationary situation. Either EGRET detected sources are special, unrepresentative objects, or their $\gamma$-ray duty cycle must be low. Indeed, strong variability at $\gamma$-ray energies is very common, and most objects have been detected in widely different intensity states. A plausible scenario for the origin of the $\gamma$-ray background is that it is caused by a mixture of inverse Compton radiation produced by LBL during strong flares (Fig. 12) and perhaps a less variable component due to the still rising part of the Compton spectrum in HBL objects (Fig. 13).

A TeV cosmic Background? The existence of a cosmic background at $\mathrm{TeV}$ energies has not yet been established. Blazars of the HBL type, especially those where the synchrotron peak is located at very high energies can produce a significant integrated flux (Fig. 13). A precise prediction is, however, difficult given the uncertainties in the amount of absorption of $\mathrm{TeV}$ photons via interaction with photons of the Infra-red background. In addition, we note that since the space density of extreme HBLs is very low (less than one object in one hundred square degrees at a radio flux of $3.5 \mathrm{mJy}$ ), the $\mathrm{TeV}$ background produced by blazars is composed of widely separated discrete sources rather than an apparently diffuse light like e.g. the cosmic X-ray background.

In all our estimations, we have assumed that the broad band SED of blazars is characterized by approximately equal power in the synchrotron and inverse Compton components. We note that an average SED, where the inverse Compton component is much more powerful than the synchrotron component, is not acceptable, as it would predict a much larger than observed background at hard-X-ray/soft Gamma ray energies (see Fig. 10). The large inverse Compton emission compared to synchrotron flux observed in some LBL sources (e.g., Ballo et al. 2002) should therefore be associated to either rare objects or transient events rather than to an average emission.

Blazars and future observatories. Figure 14 shows the observed SED of the well-known LBL blazar 3C 279, scaled down by a factor 1000, overlaid with the limiting sensitivities of the upcoming Planck and GLAST satellites and with

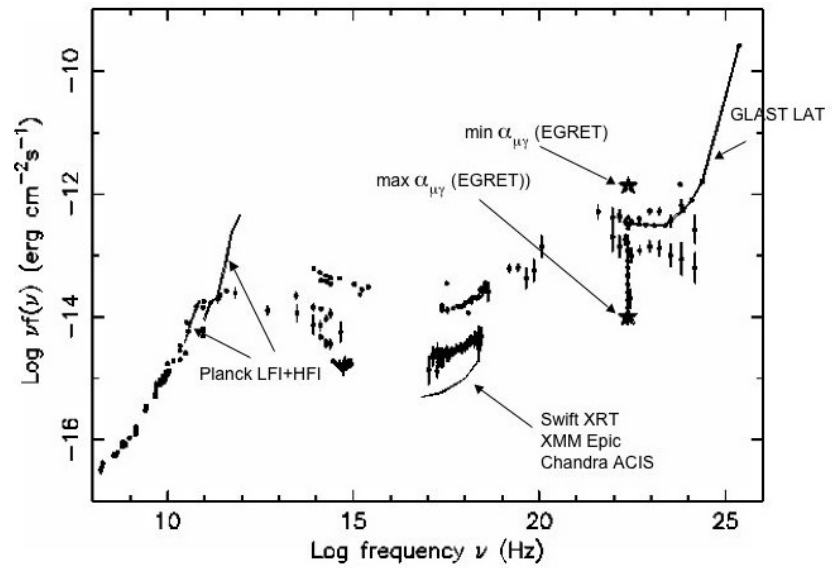

Fig. 14. The SED of the Blazar 3C 279 scaled down by a factor of 1000 ( 1 milli-3C 279) with superimposed the sensitivity of microwave (Planck LFI+HFI), X-ray (Swift XRT-XMM-Chandra) and $\gamma$-ray (GLAST-LAT) satellites. A Blazar of this flux, or about $10 \mathrm{mJy}$ at radio frequencies, would still be detected by Planck, by existing optical and X-ray satellites (Swift, XMM or Chandra) and would be above the GLAST limiting sensitivity during strong flares.

a $0.5-10 \mathrm{keV}$ sensitivity that can be reached by existing $\mathrm{X}$-ray observatories. This hypothetical $\sim 10 \mathrm{mJy}$ LBL (or 1 milli-3C 279) is at the limit of the Planck sensitivity, is detectable with a deep Swift exposure (or a less deep XMM-Newton or Chandra observation), and is detectable by GLAST during strong flares. Since the radio $\log N-\log S$ of Fig. 2 predicts a space density of $\gtrsim 5$ objects per square degree with flux above $10 \mathrm{mJy}$, the Planck mission should detect $\approx 100000-200000$ blazars in the $\approx 30000$ square degree high galactic latitude sky. A fraction of these sources will also be detected by GLAST when flaring, but exactly how many strongly depends on the duty cycle.

A deep all-sky X-ray survey to clean the data from Planck
and future CMB missions and to provide a database of
$>100000$ blazars for GLAST and other $\gamma$-ray observatories. Given the significant impact of the blazar foreground emission on the CMB power spectrum it is important to remove this contaminating component from the CMB as much as possible. One possibly efficient way to achieve this is to exploit the fact that the spectral slope distribution between microwave and soft X-ray flux of LBLs is very narrow (see Fig. 9) with a dispersion that is probably mostly due to intrinsic variability. The soft X-ray flux of LBLs (that is $>90 \%$ of the blazar population) is therefore a very good estimator of the flux at microwave frequencies and could be used to locate and remove foreground blazars from the CMB. Figures 9 and 14 imply that a hypothetical all-sky survey with limiting sensitivity of a few $10^{-15} \mathrm{erg} \mathrm{cm}^{-2} \mathrm{~s}^{-1}$ in the soft X-ray band would detect the large majority of blazars above the limiting sensitivity of Planck and therefore allow the construction of a database that includes well over 100000 blazars with flux measurements at radio, microwave, and $\mathrm{X}$-ray frequencies. Although this type of survey is currently not planned for the near future, they are clearly needed to address the previous issues. The extremely 
large sample of blazars produced by such survey could also be used to study the statistical properties of blazars in great detail, including the spatial correlation function and would provide a large number of targets for the next generation of $\gamma$-ray observatories such as AGILE, GLAST, and future instruments operating in the still poorly explored $\mathrm{MeV}$ spectral region.

Acknowledgements. This work is partly based on XMM-Newton, BeppoSAXand ROSAT X-ray archival data taken from the the ASI Science Data Center (ASDC), Frascati, Italy, and on data taken from the following on-line services: the NASA/IPAC Extragalactic Database (NED) and the Sloan Digital Sky Survey (SDSS, Data Release 3).

We are grateful to Paolo Padovani for providing DXRBs $\log N-\log S$ results in advance of publication and to Enrico Massaro for useful discussions. S.C. is supported by PRIN-MIUR under contract No. 2004027755_003.

\section{References}

Aharonian, F. A., Akhperjanian, A. G., Barrio, J. A., et al. 2002, APh, 17, 459

Bade, N., Beckmann, V., Douglas, N. G., et al. 1998, A\&A, 334, 459

Ballo, L., Maraschi, L., Tavecchio, F., et al. 2002, ApJ, 567, 50

Bennett, C. L., Bay, M., Halpern, M., et al. 2003a, ApJ, 583, 1

Bennett, C. L., Hill, R. S., Hinshaw, G., et al. 2003b, ApJS, 148, 97

Blandford, R. D., \& Rees, M. J. 1978, in Pittsburgh Conference on BL Lac Objects, ed. A. M. Wolfe (University of Pittsburgh, Pittsburgh), 328

Caccianiga, A., \& Marchã, M. J. 2004, MNRAS, 348, 937

Caccianiga, A., Marchã, M. J., Antón, S., Mack, K.-H., \& Neeser, M. J. 2002, MNRAS, 329, 877

Cavazzuti, E., et al. 2005, in preparation

Condon, J. J., Cotton, W. D., Greisen, E. W., et al. 1998, AJ, 115, 1693

di Serego-Alighieri, S., Danziger, I. J., Morganti, R., \& Tadhunter, C. N. 1994, MNRAS, 269, 998

Fossati, G., Celotti, A., Ghisellini, G., \& Maraschi, L. 1997, MNRAS, 289, 136

Galbiati, E., Caccianiga, A., Maccacaro, T., et al. 2005, A\&A, 430, 927

Ghisellini, G., Celotti, A., Fossati, G., Maraschi, L., \& Comastri, A. 1998, MNRAS, 301, 451

Giacconi, R., Gursky, H., Paolini, F., \& Rossi, B. 1962, Phys. Rev. Lett., 9, 439

Giommi, P., \& Colafrancesco, S. 2004, A\&A, 414, 7

Giommi, P., Menna, M. T., \& Padovani, P. 1999, MNRAS, 310, 465

Giommi, P., Capalbi, M., Fiocchi, M., et al. 2002a, in Blazar Astrophysics with BeppoSAX and Other Observatories, ed. P. Giommi, E. Massaro, \& G. Palumbo, 63

Giommi, P., Padovani, P., Perri, M., Landt, H., \& Perlman, E. 2002b, in Blazar Astrophysics with BeppoSAX and Other Observatories, ed. P. Giommi, E. Massaro, \& G. Palumbo, 133

Giommi, P., Perri, M., Piranomonte, S., \& Padovani, P. 2002c, in Blazar Astrophysics with BeppoSAX and Other Observatories, ed. P. Giommi, E. Massaro, \& G. Palumbo, 123

Giommi, P., Piranomonte, S., Perri, M., \& Padovani, P. 2005, A\&A, 434, 385

Gonzales-Nuevo, J., Toffolatti, L., \& Argueso, F. 2005, ApJ, 621, 1

Gruber, D. E., Matteson, J. L., Peterson, L. E., \& Jung, G. V. 1999, ApJ, 520, 124
Hartman, R. C., Bertsch, D. L., Bloom, S. D., et al. 1999, ApJS, 123, 79

Kappadath, S. C. 1998, Ph.D. Thesis, University of New Hampshire, USA

Kushino, A., Ishisaki, Y., Morita, U., et al. 2002, PASJ, 54, 327

Landt, H., Padovani, P., Perlman, E. S., et al. 2001, MNRAS, 323, 757

Loan, A. J., Wall, J., \& Lahav, O. 1997, MNRAS, 286, 994

Lumb, D. H., Warwick, R. S., Page, M., \& De Luca, A. 2002, A\&A, 389, 93

Marshall, F. E., Boldt, E. A., Holt, S. S., et al. 1980, ApJ, 235, 4

Mather, J., Fixsen, D., Shafer, R. A., Mosier, C., \& Wilkinson, D. T. 1999, ApJ, 512, 511

Mattox, J., Schachter, J., Molnar, L., Hartman, R., \& Patnaik, A. 1997, ApJ, 481, 95

McLean, B., Greene, G. R., Lattanzi, M. G., \& Pirenne, B. 2000, in ADASS IX, ed. C. Veillet, D. Crabtree, \& N. Manset, ASP Conf. Ser., 216, 145

Moretti, A., Campana, S., Lazzati, D., \& Tagliaferri, G. 2003, ApJ, 588, 696

Nieppola, E., Tornikoski, M., \& Valtaoja, E. 2005, A\&A, submitted

Padovani, P. 2002, in Blazar Astrophysics with BeppoSAX and Other Observatories, ed. P. Giommi, E. Massaro, \& G. Palumbo, 101

Padovani, P., Ghisellini, G., Fabian, A., \& Celotti, A. 1993, MNRAS, 260, L21

Padovani, P., \& Giommi, P. 1995, ApJ, 444, 567

Padovani, P., Perlman, E., Landt, H., Giommi, P., \& Perri, M. 2003a, ApJ, 58, 128

Padovani, P., Perlman, E., Landt, H., Giommi, P., \& Perri, M. 2003b, ApJ, 588, 128

Padovani, P., Giommi, P., et al. 2005, in preparation

Peebles, P. 1980, in The Large Scale Sturcture of the Universe, ed. P. U. P. Priceton

Peebles, P. 1997, ApJ, 483, L1

Perlman, E. S., Padovani, P., Giommi, P., et al. 1998, AJ, 115, 1253

Perri, M., \& Giommi, P. 2000, MNRAS, 362, L61

Piranomonte, S., Perri, M., Giommi, P., Padovani, P., \& Landt, H. 2004, A\&A, submitted

Rector, T. A., Stocke, J. T., Perlman, E. S., Morris, S. L., \& Gioia, I. M. 2000, AJ, 120, 1626

Rosati, P., Tozzi, P., Giacconi, R., et al. 2002, ApJ, 566, 667

Schmidt, M. 1963, Nature, 197, 1040

Scott, D., \& White, M. 1999, A\&A, 346, 1

Sreekumar, P., Bertsch, D. L., Dingus, B. L., et al. 1998, ApJ, 494, 523

Stoughton, C., Lupton, R. H., Bernardi, M., et al. 2002, AJ, 123, 485

Strong, A., Moskalenko, I., \& Reimer, O. 2004, ApJ, 613, 956

Strüder, L., Briel, U., Dennerl, K., et al. 2001, A\&A, 365, L18

Tegmark, M., \& Efstathiou, G. 1996, MNRAS, 281, 1297

Toffolatti, L., Argueso Gomez, F., de Zotti, G., et al. 1998, MNRAS, 297, 117

Turriziani, S., Giommi, P., \& Cavazzuti, E. 2005, in preparation

Urry, C. M., \& Padovani, P. 1995, PASP, 107, 803

Vecchi, A., Molendi, S., Guainazzi, M., Fiore, F., \& Parmar, A. N. 1999, A\&A, 349, L73

Voges, W., Aschenbach, B., Boller, T., et al. 1999, A\&A, 349, 389

Wall, J. V., Jackson, C. A., Shaver, P. A., Hook, I. M., \& Kellermann, K. I. 2004, A\&A, submitted

Wall, J. V., \& Peacock, J. A. 1985, MNRAS, 216, 173

White, N. E., Giommi, P., \& Angelini, L. 1995,

http://wgacat.gsfc.nasa.gov

York, D. G., Adelman, J., Anderson, J. E., J., et al. 2000, AJ, 120, 1579 


\section{Online Material}


P. Giommi et al.: Non-thermal cosmic backgrounds from blazars, Online Material $p 2$

Table 4. The list and properties of all WMAP-detected blazars associated to EGRET $\gamma$-ray sources.

\begin{tabular}{|c|c|c|c|c|c|c|c|c|c|}
\hline Blazar name & $\begin{array}{c}\text { RA } \\
\text { J2000.0 }\end{array}$ & $\begin{array}{l}\text { Dec } \\
\text { J2000.0 } \\
\text { (3) }\end{array}$ & $\begin{array}{c}\text { Radio flux } \\
5 \mathrm{GHz} \\
\mathrm{Jy} \\
(4)\end{array}$ & $\begin{array}{c}\text { WMAP flux } \\
94 \mathrm{GHz} \\
\text { Jy } \\
(5)\end{array}$ & $\begin{array}{c}\text { EGRET flux } \\
>100 \mathrm{MeV} \\
10^{-8} \mathrm{ph} \mathrm{cm}^{-2} \mathrm{~s}^{-1} \\
\text { (6) }\end{array}$ & $\overline{\alpha_{\mu \gamma}}$ & $\begin{array}{c}\text { Duty } \\
\text { cycle } \\
\% \\
(8)\end{array}$ & $\begin{array}{c}\text { EGRET } \\
\text { name } \\
\text { 3EG J } \\
(9)\end{array}$ & $\begin{array}{c}\text { WMAP } \\
\text { catalog } \\
\text { number } \\
(10)\end{array}$ \\
\hline $4 \mathrm{C} 15.05$ & 020450.3 & 151410 & 3.073 & $1.6^{*}$ & $9-53$ & $0.846-0.914$ & $2-12$ & $0204+1458$ & 092 \\
\hline 1Jy0208-512 & 021046.2 & -510102 & 3.198 & 1.8 & $35-134$ & $0.816-0.867$ & $1-4$ & $0210-5055$ & 158 \\
\hline B2 $0234+28$ & 023752.3 & 284808 & 2.794 & $2.1^{*}$ & $11-31$ & $0.877-0.917$ & $5-13$ & $0239+2815$ & 093 \\
\hline CTA26 & 033930.8 & -014635 & 3.014 & 3.2 & $13-178$ & $0.827-0.926$ & $1-17$ & 0340-0201 & 106 \\
\hline PKS 0420-01 & 042315.7 & -012032 & 4.357 & 3.9 & $9.3-64.2$ & $0.873-0.946$ & $4-29$ & $0422-0102$ & 110 \\
\hline 1Jy0454-463 & 045550.7 & -461559 & 1.653 & 3.8 & $5.5-22.8$ & $0.911-0.966$ & $11-47$ & $0458-4635$ & 151 \\
\hline 1Jy0454-234 & 045703.1 & -232451 & 1.863 & 2.7 & $8.1-14.7$ & $0.915-0.938$ & $13-23$ & $0456-2338$ & 128 \\
\hline PKS 0506-61 & 050644.0 & -610940 & 1.211 & $1.1^{*}$ & $6-29$ & $0.855-0.915$ & $3-13$ & $0512-6150$ & 154 \\
\hline 1Jy0537-441 & 053851.3 & -440511 & 4.805 & 6.7 & $16.5-91.1$ & $0.880-0.945$ & $5-28$ & 0540-4402 & 148 \\
\hline PKS $0735+178$ & 073807.3 & 174218 & 1.812 & $1.7^{*}$ & $15-29$ & $0.872-0.896$ & $4-8$ & $0737+1721$ & 113 \\
\hline B2 $0827+24$ & 083052.0 & 241057 & 0.886 & $2.6^{*}$ & $16-111$ & $0.837-0.911$ & $2-11$ & $0829+2413$ & 112 \\
\hline $\mathrm{S} 50836+710$ & 084124.4 & 705340 & 2.342 & $1.2^{*}$ & $9-33$ & $0.854-0.903$ & $2-9$ & $0845+7049$ & 089 \\
\hline OJ 287 & 085448.8 & 200630 & 2.908 & 2.5 & $9.7-15.8$ & $0.910-0.928$ & $11-18$ & $0853+1941$ & 115 \\
\hline 4C 29.45 & 115931.7 & 291443 & 1.461 & 2.1 & $7.5-163.2$ & $0.814-0.931$ & $1-19$ & $1200+2847$ & 111 \\
\hline PKS1221-82 & 122454.3 & -831310 & 0.797 & $1.2^{*}$ & $11-36$ & $0.850-0.895$ & $2-7$ & $1249-8330$ & 178 \\
\hline 1Jy $1226+023$ & 122906.3 & 020304 & 36.923 & 9.0 & $8.5-48.3$ & $0.916-0.982$ & $13-73$ & $1229+0210$ & 170 \\
\hline 3C 279 & 125611.0 & -054719 & 11.192 & 19.0 & $15-250$ & $0.882-1.000$ & $5-100$ & $1255-0549$ & 181 \\
\hline PKS 1313-333 & 131607.9 & -333859 & 1.093 & $1.3^{*}$ & $15-32$ & $0.858-0.887$ & $3-6$ & $1314-3431$ & 182 \\
\hline 1Jy1406-076 & 140856.4 & -075225 & 1.080 & $1.7^{*}$ & $10-128$ & $0.815-0.912$ & $1-12$ & $1409-0745$ & 203 \\
\hline 1Jy1424-418 & 142756.2 & -420619 & 2.597 & $1.5^{*}$ & $12-55$ & $0.842-0.901$ & $2-9$ & $1429-4217$ & 191 \\
\hline 1Jy1510-089 & 151250.4 & -090600 & 3.080 & 1.7 & $12.6-49.4$ & $0.851-0.903$ & $2-9$ & $1512-0849$ & 207 \\
\hline 1Jy1606+106 & 160846.0 & 102907 & 1.412 & 3.1 & $21.0-62.4$ & $0.865-0.907$ & $3-10$ & $1608+1055$ & 009 \\
\hline DA 406 & 161340.9 & 341246 & 2.324 & 1.4 & $19-68.9$ & $0.831-0.880$ & $1-5$ & $1614+3424$ & 023 \\
\hline 4C 38.41 & 163515.4 & 380804 & 3.221 & 4.2 & $31.8-107.5$ & $0.856-0.902$ & $3-9$ & $1635+3813$ & 033 \\
\hline PMNJ1703-6212 & 170336.2 & -621239 & 0.616 & $1.9^{*}$ & $14-53$ & $0.853-0.904$ & $2-9$ & $1659-6251^{b}$ & 198 \\
\hline S41739+522 & 174036.9 & 521142 & 1.699 & $1.2^{*}$ & $10-45$ & $0.842-0.899$ & $2-8$ & $1738+5203$ & 048 \\
\hline PKS $1814-63^{c}$ & 181934.9 & -634547 & 4.506 & $1.3^{*}$ & $14-27$ & $0.864-0.889$ & $3-6$ & $1813-6419$ & 200 \\
\hline PMNJ1923-2104 & 192332.1 & -210433 & 2.885 & $2.1^{*}$ & $29^{* *}$ & 0.880 & 5 & 1921-2015 & 008 \\
\hline PKS 2052-47 & 205615.5 & -471437 & 2.026 & $1.3^{*}$ & $9-35$ & $0.854-0.906$ & $3-10$ & $2055-4716$ & 208 \\
\hline BL Lac & 220243.2 & 421639 & 2.940 & $3.8^{*}$ & $9-40$ & $0.890-0.947$ & $7-29$ & $2202+4217$ & 058 \\
\hline PKS2209+236 & 221205.9 & 235539 & 1.123 & $1.3^{*}$ & $7-46$ & $0.844-0.916$ & $2-13$ & $2209+2401$ & 050 \\
\hline CTA102 & 223236.3 & 114350 & 3.967 & 3.1 & $12.1-51.6$ & $0.873-0.928$ & $4-18$ & $2232+1147$ & 047 \\
\hline $1 \mathrm{Jy} 2251+158$ & 225357.6 & 160852 & 14.468 & 5.9 & $24.6-116.1$ & $0.866-0.925$ & $3-16$ & $2254+1601$ & 055 \\
\hline $1 \mathrm{Jy} 2351+456$ & 235421.6 & 455303 & 1.127 & $1.7^{*}$ & $12-43$ & $0.874-0.923$ & $4-15$ & $2358+4604$ & 074 \\
\hline
\end{tabular}

* Not detected by WMAP in the W-band; flux taken from $90 \mathrm{GHz}$ measurements.

${ }^{* *}$ Detected only once by EGRET reported in NED or extrapolated from lower (K,Ka,Q, V-Band) WMAP channels.

${ }^{a}$ This source does not have an optical spectroscopic identification yet. However, the radio SED is very flat, typical of blazars. We propose this as a blazar candidate and associate it with the EGRET source 3EG J1249-8330 (previously tentatively associated to PMNJ1249-8303 by Mattox et al. 1997).

${ }^{b}$ This EGRET detection has also been associated to the bright radio source PMN J1647-6437 (Mattox et al. 1997) which is further away from the $\gamma$-ray position.

${ }^{c}$ This source is a radio galaxy with steep radio spectrum at centimeter wavelengths. At microwave frequencies where WMAP is sensitive its spectrum is flat and indistinguishable from a that of a blazar. 